www.nature.com/ejhg

\title{
ARTICLE
}

\section{Combined high resolution linkage and association mapping of quantitative trait loci}

\author{
Ruzong Fan ${ }^{*, 1,2}$ and Momiao Xiong ${ }^{3}$
}

${ }^{1}$ Department of Statistics, The Texas A\&M University, 447 Blocker Building, College Station, Texas 77843-3143, USA; ${ }^{2}$ Institute of Medical Biometry, Informatics and Epidemiology, University of Bonn, Sigmund Freud Strasse 25, D-53105 Bonn, Germany; ${ }^{3}$ Human Genetics Center, University of Texas - Houston, P.O. Box 20334, Houston, Texas 77225, USA

In this paper, we investigate variance component models of both linkage analysis and high resolution linkage disequilibrium (LD) mapping for quantitative trait loci (QTL). The models are based on both family pedigree and population data. We consider likelihoods which utilize flanking marker information, and carry out an analysis of model building and parameter estimations. The likelihoods jointly include recombination fractions, LD coefficients, the average allele substitution effect and allele dominant effect as parameters. Hence, the model simultaneously takes care of the linkage, LD or association and the effects of the putative trait locus. The models clearly demonstrate that linkage analysis and LD mapping are complementary, not exclusive, methods for QTL mapping. By power calculations and comparisons, we show the advantages of the proposed method: (1) population data can provide information for LD mapping, and family pedigree data can provide information for both linkage analysis and LD mapping; (2) using family pedigree data and a sparse marker map, one may investigate the prior suggestive linkage between trait locus and markers to obtain low resolution of the trait loci, because linkage analysis can locate a broad candidate region; (3) with the prior knowledge of suggestive linkage from linkage analysis, both population and family pedigree data can be used simultaneously in high resolution LD mapping based on a dense marker map, since LD mapping can increase the resolution for candidate regions; (4) models of high resolution LD mappings using two flanking markers have higher power than that of models of using only one marker in the analysis; (5) excluding the dominant variance from the analysis when it does exist would lose power; (6) by performing linkage interval mappings, one may get higher power than by using only one marker in the analysis.

European Journal of Human Genetics (2002) 11, 125 -137. doi:10.1038/sj.ejhg.5200941

Keywords: variance component models; linkage interval mapping; linkage disequilibrium mapping; QTL

\section{Introduction}

Twenty years ago, variations in human DNA were recognized as genetic markers in linkage study. ${ }^{1}$ After that, the advances in molecular biology and computational technology have led to mapping several human inher-

${ }^{*}$ Correspondence: Dr Ruzong Fan; Department of Statistics, The Texas A\&M University, 447 Blocker Building, College Station, Texas 77843-3143, USA. Tel: 979-845-3156; Fax: 979-845-3144; E-mail: rfan@stat.tamu.edu Received 4 June 2002; revised 14 October 2002; accepted 21 November 2002 ited disease genes. Using restriction fragment length polymorphism (RFLP) markers and polymorphic microsatellite loci, linkage analysis and positional cloning have been used successfully in mapping the chromosome locations of Mendelian disease genes. The success mainly depends on one premise that the disease genes of Mendelian traits have a large effect on the phenotypes. ${ }^{2}$ In fact, there is usually a one-to-one correspondence between disease gene genotypes and the disease phenotype for Mendelian traits. Moreover, the correlations 
between genotypes and phenotype of Mendelian traits are strong. Given sufficient family data, Mendelian traits can be mapped with high probability by linkage analysis.

With the encouragement of successful mapping Mendelian trait genes, there has been growing interests and endeavors in the study of complex traits such as asthma and diabetes. For complex diseases, the inheritance patterns and phenotype definitions as with genetic etiology are much more complex. The trait/affection status is usually a continuous variable. ${ }^{3}$ The mapping of complex disease genes is much harder. Novel statistical methods such as both linkage analysis and linkage disequilibrium (LD) mapping or association study are needed in dissecting complex traits. As very dense marker maps such as single nucleotide polymorphism (SNP) are available, ${ }^{4}$ both linkage analysis and association study are utilized simultaneously for mapping complex disease loci. ${ }^{5,6}$ Almasy et $a l^{7}$ and Fulker et $a l^{8}$ proposed to use combined linkage and association analysis for quantitative trait loci (QTL). Sham et $a l^{9}$ studied the power of linkage versus association analysis of quantitative traits by analytically calculating non-centrality parameters of test statistics. Abecasis et $a^{10-12}$ proposed test statistics of association studies for quantitative traits in nuclear families, general pedigrees, and selected samples. Cardon ${ }^{13}$ studied a sibpair regression model of $\mathrm{LD}$ for quantitative traits. All these researches concentrated on family data which include sib-pairs, and used only one marker in analysis. In Fan and Xiong, ${ }^{14}$ we proposed a linear regression method of high resolution mapping of quantitative trait loci by LD mapping analysis. The method is based on population data. Using two flanking markers, the regression models have higher power than that of models using only one marker. ${ }^{14}$

It is well-known that family pedigree data can be used in both linkage analysis and association study, and population data can be used in association study. Hence, it is necessary to consider a method to combine both population data and family pedigree data in the analysis. In this paper, we propose to perform both linkage analysis and high resolution LD mapping for QTL based on combined family and population data. Linkage interval mapping is based on family data, and LD mapping is based on both family pedigree and population data. Based on variance component models, we construct likelihood to analyse family and population data in Section of Models. Then, we discuss the parameter estimations and regression coefficients. The linkage information, i.e., recombination fractions, is contained in the variance-covariance matrix, and the association information, i.e., the LD coefficients, is contained in the mean parameters or the regression coefficients. We calculate the non-centrality parameters for association study and linkage analysis, respectively. Using the noncentrality parameters, we perform power calculations and comparisons. The technical details to calculate the regression coefficients, parameters, non-centrality parameters are left in the Appendixes.

\section{Models}

Consider a quantitative trait locus $Q$ which has two alleles $Q_{1}$ and $Q_{2}$. Suppose that the allele frequencies of $Q_{1}$ and $Q_{2}$ are $q_{1}$ and $q_{2}$, respectively. Assume that two markers $A$ and $B$ flank the trait locus $Q$ in an order of $A Q B$. Marker $A$ has two alleles $A$ and $a$ with frequencies $P_{A}$ and $P_{a}$, respectively. Marker $B$ has two alleles $B$ and $b$ with frequencies $P_{B}$ and $P_{b}$, respectively. For a nuclear family of $k$ children and two parents, let us denote their quantitative traits by a vector $\mathrm{y}=\left(y_{f}, y_{m}, y_{1}, \cdots, y_{k}\right)^{\tau}$, genotypes at marker $A$ by a vector $\left(A_{f}, A_{m}, A_{1}, \cdots, A_{k}\right)^{\tau}$, and genotypes at marker $B$ by a vector $\left(B_{f}, B_{m}, B_{1}, \cdots, B_{k}\right)^{\tau}$. Here $y_{f}$ is the trait value of the father, $A_{f}$ is the genotype of the father at marker $A$, and $B_{f}$ is the genotype of the father at marker $B$. Other notations are defined, similarly, for the mother with subscript $m$ and for the $i$-th child with subscript $i$. The log-likelihood is defined by $L=\frac{k+2}{2} \log (2 \pi) \quad \frac{1}{2} \log |\Sigma| \quad \frac{1}{2}(\mathbf{y} \quad X \mu)^{\tau} \Sigma^{1}$ (y $X \mu$ ). The notations of the log-likelihood are defined as follows. For the mean component $X \mu$, we consider the following regression equation such as model (1) in Fan and Xiong ${ }^{14}$

$$
y_{i}=\beta+\omega_{i} \gamma+x_{A i} \alpha_{A}+x_{B i} \alpha_{B}+z_{A i} \delta_{A}+z_{B i} \delta_{B}+G_{i}+e_{i},
$$

where $\beta$ is overall mean, $w_{i}$ is a row vector of covariates such as gender and age, $\gamma$ is a column vector of regression coefficients for the covariates $w_{i}, G_{i}$ is polygenic effect, $e_{i}$ is error term. Assume that $G_{i}$ is normal $N\left(0, \sigma_{G}^{2}\right)$, and $e_{i}$ is normal $N\left(0, \sigma_{e}^{2}\right)$. Moreover, $G_{i}$ and $e_{i}$ are independent. $x_{A i}$, $x_{B i}, Z_{A i}$ and $z_{B i}$ are dummy variables defined by

$$
\begin{aligned}
& x_{A i}=\left\{\begin{array}{lll}
2 P_{a} & \text { if } & A_{i}=A A \\
P_{a} P_{A} & \text { if } & A_{i}=A a \\
2 P_{A} & \text { if } & A_{i}=a a
\end{array} \quad Z_{A i}=\left\{\begin{array}{ccc}
P_{a}^{2} & \text { if } & A_{i}=A A \\
P_{a} P_{A} & \text { if } & A_{i}=A a \\
P_{A}^{2} & \text { if } & A_{i}=a a
\end{array}\right.\right. \\
& x_{B i}=\left\{\begin{array}{ccc}
2 P_{b} & \text { if } & B_{i}=B B \\
P_{b} P_{B} & \text { if } & B_{i}=B b \\
2 P_{B} & \text { if } & B_{i}=b b
\end{array} \quad_{B i}^{2}=\left\{\begin{array}{ccc}
P_{b} & \text { if } & B_{i}=B B \\
P_{b} P_{B} & \text { if } & B_{i}=B b . \\
P_{B}^{2} & \text { if } & B_{i}=b b
\end{array}\right.\right.
\end{aligned}
$$

$\alpha_{A}, \alpha_{B}, \delta_{A}$, and $\delta_{B}$ are regression coefficients of the dummy variables $x_{A i}, x_{B i}, z_{A i}$ and $z_{B i}$.

The model matrix $X$ is defined by

$$
X=\left\{\begin{array}{cccccc}
1 & w_{f} & x_{A f} & x_{B f} & Z_{A f} & Z_{B f} \\
1 & w_{m} & x_{A m} & x_{B m} & Z_{A m} & Z_{B m} \\
1 & w_{1} & x_{A 1} & x_{B 1} & Z_{A 1} & Z_{B 1} \\
\vdots & \vdots & \vdots & \vdots & \vdots & \\
1 & w_{k} & x_{A k} & x_{B k} & Z_{A k} & Z_{B k}
\end{array}\right\}=\left(\begin{array}{c}
X_{f}^{\tau} \\
X_{m}^{\tau} \\
X_{1}^{\tau} \\
\vdots \\
X_{k}^{\tau}
\end{array}\right),
$$

and $\mu=\left(\beta, \gamma^{\tau}, \alpha_{A}, \alpha_{B}, \delta_{A}, \delta_{B}\right)^{\tau}$ is a vector of regression coefficients. $\Sigma$ is a $(k+2) \times(k+2)$ 
variance-covariance matrix defined as

$$
\Sigma=\left(\begin{array}{cccccc}
1 & 0 & \rho_{0} & \rho_{0} & \cdots & \rho_{0} \\
0 & 1 & \rho_{0} & \rho_{0} & \cdots & \rho_{0} \\
\rho_{0} & \rho_{0} & 1 & \rho_{12} & \cdots & \rho_{1 k} \\
\rho_{0} & \rho_{0} & \rho_{21} & 1 & \cdots & \rho_{2 k} \\
\vdots & \vdots & \vdots & \vdots & \cdots & \vdots \\
\rho_{0} & \rho_{0} & \rho_{k 1} & \rho_{k 2} & \cdots & 1
\end{array}\right) \sigma^{2} \text {, where } \sigma^{2}=
$$

$\sigma_{g}^{2}+\sigma_{G}^{2}+\sigma_{e}^{2}, \sigma_{g}^{2}$ is variance explained by the putative QTL $Q$ $\sigma_{G}^{2}$ is polygenic variance, and $\sigma_{e}^{2}$ is error variance. The genetic variances $\sigma_{g}^{2}=\sigma_{g a}^{2}+\sigma_{g d}^{2}$ and $\sigma_{G}^{2}=\sigma_{G a}^{2}+\sigma_{G d}^{2}$ are decomposed into additive and dominant components. $\rho_{0}=\left(\sigma_{g a}^{2}+\sigma_{G a}^{2}\right) /\left(2 \sigma^{2}\right)$ is correlation between parents and children, $\rho_{i j}=\left(\pi_{i j Q} \sigma_{g a}^{2}+\Delta_{i j Q} \sigma_{g d}^{2}+\sigma_{G a}^{2} / 2+\sigma_{G d}^{2} / 4\right) / \sigma^{2}$ is correlation between the $i$-th child and the $j$-th child, $\pi_{i j Q}$ is the proportion of alleles shared identical by descent (IBD) at QTL $Q$ by the $i$-th child and the $j$-th child, and $\Delta_{i j Q}$ is the probability that both alleles at QTL $Q$ shared by the $i$-th child and the $j$-th child are IBD.

For population data, an intuitive rationale of regression model (1) is given in Fan and Xiong ${ }^{14}$. In general, one can construct a variance-covariance matrix for any type of pedigree in a similar way as above. Assume that there are two independent sub-samples of data: (1) population data: $n$ independent individuals; (2) family data: $I-n(I>n)$ independent families. Let us list the log-likelihood of the $n$ independent individuals by $L_{1}, \cdots, L_{n}$, and the likelihood of the $I-n$ families by $L_{n+1}, \cdots, L_{I}$. Then the overall log-likelihood is $L=\sum_{i=1}^{I} L_{i}$. The unknown parameters are $\mu=$ $\left(\beta, \gamma, \alpha_{A}, \alpha_{B}, \delta_{A}, \delta_{B}\right)^{\tau}, \sigma_{g a}^{2}, \sigma_{g d}^{2}, \sigma_{G a}^{2}, \sigma_{G d}^{2}$, and $\sigma_{e}^{2}$. Using the likelihood ratio tests, one may test statistical significance of the parameters of interest.

\section{Parameter estimations and regression coefficients}

Regression coefficients Let $\mu_{i j}$ be the effect of genotype $Q_{i} Q_{j}, i, j=1,2, \mu_{12}=\mu_{21}$. Denote the population effect mean by $\mu=\mu_{11} q_{1}^{2}+2 \mu_{12} q_{1} q_{2}+\mu_{22} q_{2}^{2}$ and define $\alpha_{Q}=q_{1} \mu_{11}$ $+\left(q_{2}-q_{1}\right) \mu_{12}-q_{2} \mu_{22}, \delta_{Q}=2 \mu_{12}-\mu_{11}-\mu_{22}$. If $\mu_{11}=a, \mu_{12}=d$, and $\mu_{22}=-a$ as in the traditional quantitative genetics, ${ }^{15} \alpha_{Q}=$ $a+\left(q_{2}-q_{1}\right) d$ is the average allele substitution effect, and $\delta_{Q}$ $=2 d$ characterizes the dominant effect. In general, one may define $a=\mu_{11}-\left(\mu_{11}+\mu_{22}\right) / 2$ and $d=\mu_{12}-\left(\mu_{11}+\mu_{22}\right) / 2$. It is well known that the additive variance $\sigma_{g a}^{2}=2 q_{1} q_{2} \alpha_{Q}^{2}$ and the dominant variance $\sigma_{g d}^{2}=\left(q_{1} q_{2}\right)^{2} \delta_{Q}^{2}$. A true random effect model describing the trait value is $y_{i}=\beta+w_{i} \gamma+g_{i}+G_{i}+e_{i}$ where

$$
g_{i}=\left\{\begin{array}{lll}
\mu_{11} & \text { for genotype } & Q_{1} Q_{1} \\
\mu_{12} & \text { for genotype } & Q_{1} Q_{2} \\
\mu_{22} & \text { for genotype } & Q_{2} Q_{2}
\end{array}\right.
$$

Denote LD coefficient between trait locus $Q$ and marker $A$ by $D_{A Q}=P\left(A Q_{1}\right)-q_{1} P_{A}$, LD coefficient between trait locus $Q$ and marker $B$ by $D_{Q B}=P\left(B Q_{1}\right)-q_{1} P_{B}$, and LD coefficient between marker $A$ and marker $B$ by $D_{A B}=P(A B)-P_{A} P_{B}$. Let the additive and dominant variance-covariance matrices be

$$
V_{A}=\left(\begin{array}{cc}
2 P_{a} P_{A} & 2 D_{A B} \\
2 D_{A B} & 2 P_{b} P_{B}
\end{array}\right), \text { and } V_{D}=\left(\begin{array}{cc}
P_{a}^{2} P_{A}^{2} & D_{A B}^{2} \\
D_{A B}^{2} & P_{b}^{2} P_{B}^{2}
\end{array}\right)
$$

Moreover, let us denote three ratios $D_{A B}^{2} /\left(P_{a} P_{A} P_{b} P_{B}\right)=$ $R_{A B}^{2}, D_{A Q}^{2} /\left(P_{a} P_{A} q_{1} q_{2}\right)=R_{A Q}^{2}$, and $D_{Q B}^{2} /\left(q_{1} q_{2} P_{b} P_{B}\right)=R_{Q B}^{2}$. As in Appendix $\mathrm{B}^{14}$ we can show that the coefficients of regression equation (1) are given by

$$
\begin{aligned}
& \left(\begin{array}{c}
\alpha_{A} \\
\alpha_{B}
\end{array}\right)=V_{A}^{1}\left(\begin{array}{c}
2 D_{A Q} \\
2 D_{Q B}
\end{array}\right) \alpha_{Q}=\left(\begin{array}{c}
\frac{R_{A Q} R_{A B} R_{Q B}}{\sqrt{P_{A} P_{a}}} \\
\frac{R_{Q B} R_{A B} R_{A Q}}{\sqrt{P_{B} P_{b}}}
\end{array}\right) \frac{\sqrt{q_{1} q_{2}} \alpha_{Q}}{1 R_{A B}^{2}}, \\
& \left(\begin{array}{l}
\delta_{A} \\
\delta_{B}
\end{array}\right)=V_{D}{ }^{1}\left(\begin{array}{l}
D_{A Q}^{2} \\
D_{Q B}^{2}
\end{array}\right) \delta_{Q}=\left(\begin{array}{c}
\frac{R_{A Q}^{2} R_{A B}^{2} R_{Q B}^{2}}{P_{A} P_{a}} \\
\frac{R_{Q B}^{2} R_{A B}^{2} R_{A Q}^{2}}{P_{B} P_{b}}
\end{array}\right) \frac{q_{1} q_{2} \delta_{Q}}{1} R_{A B}^{4} .
\end{aligned}
$$

Parameters of variance-covariances Denote the recombination fraction between trait locus $Q$ and marker $A$ by $\theta_{A Q}$, the recombination fraction between trait locus $Q$ and marker $B$ by $\theta_{Q B}$, and the recombination fraction between marker $A$ and marker $B$ by $\theta_{A B}$. Fulker and Cardon ${ }^{16}$ proposed to estimate the proportion $\pi_{i j Q}$ of allele IBD at putative QTL $Q$ for a sib-pair $i$ and $j$ by $\hat{\pi}_{i j Q}=\mathrm{E}\left(\pi_{i j Q} \mid \pi_{i j A}, \pi_{i j B}\right)=\alpha_{\pi}+\beta_{\pi A} \pi_{i j A}+$ $\beta_{\pi B} \pi_{i j B}$ where $\pi_{i j A}$ and $\pi_{i j B}$ are the IBD proportions of alleles shared at the marker $A$ and marker $B$, respectively. The coefficients $\alpha_{\pi}, \beta_{\pi A}$ and $\beta_{\pi B}$ are given by

$$
\begin{aligned}
& \beta_{\pi A}=\frac{\left(\begin{array}{llll}
1 & 2 \theta_{A Q}
\end{array}\right)^{2}\left(\begin{array}{ll}
1 & 2 \theta_{A B}
\end{array}\right)^{2}\left(\begin{array}{ll}
1 & 2 \theta_{Q B}
\end{array}\right)^{2}}{1},
\end{aligned}
$$

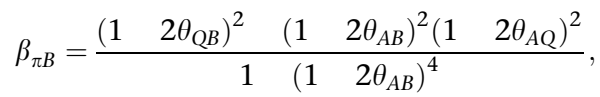

$$
\begin{aligned}
& \alpha_{\pi}=\frac{1 \quad \beta_{\pi A} \quad \beta_{\pi B}}{2} .
\end{aligned}
$$

Let $\Delta_{i j A}, \Delta_{i j B}$ be the probability of sharing two alleles IBD at markers $A$ and $B$ for a pair of sibs, respectively. In Fan, ${ }^{17}$ we proposed to estimate $\Delta_{i j Q}$ by equation $\hat{\Delta}_{i j Q}=\alpha+\beta_{A} \pi_{i j A}+$ $\beta_{B} \pi_{i j B}+r_{A} \Delta_{i j A}+r_{B} \Delta_{i j B}$. Under the assumption of no interference, the coefficients are as follows $\left(\mathrm{Fan}^{17}\right)$ :

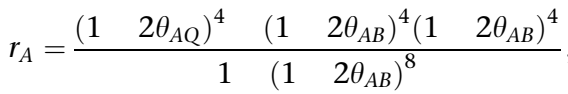

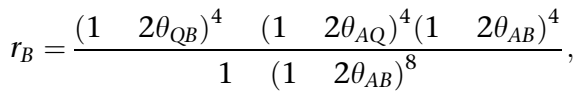

$$
\begin{aligned}
& \beta_{A}=\beta_{\pi A} \quad r_{A}, \beta_{B}=\beta_{\pi B} \quad r_{B},
\end{aligned}
$$

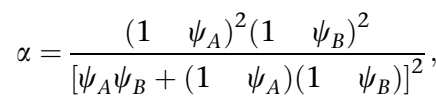

where $\psi_{A}=\theta_{A Q}^{2}+\left(\begin{array}{ll}1 & \theta_{A Q}\end{array}\right)^{2}$ and $\psi_{B}=\theta_{Q B}^{2}+\left(\begin{array}{ll}1 & \theta_{Q B}\end{array}\right)^{2}$. Assuming that the positions of marker $A$ and marker $B$ are known, $\theta_{A B}$ can be calculated through Haldane's map function. Then only one of $\theta_{A Q}$ and $\theta_{Q B}$ is unknown since the 
other can be calculated through Trow's formula. ${ }^{18}$ For general relatives $i$ and $j$, Almasy and Blangero ${ }^{19}$ proposed an algorithm to calculate the proportion $\pi_{i j Q}$ of allele IBD at putative QTL $Q$ and the expected probability $\Delta_{i j Q}$ that both alleles at QTL $Q$ are IBD. In Fan, ${ }^{17}$ we derived formulas to calculate the covariances of trait values for a few types of relatives directly without performing matrix operations.

Association and linkage studies From equations (3) and (4), we can see that the coefficients of LD (i.e., $D_{A Q}$ and $D_{Q B}$ ) and gene effects (i.e., $\alpha_{Q}$ and $\delta_{Q}$ ) are contained in the regression coefficients. Moreover, we show in the above paragraph that the linkage parameters (i.e., recombination fractions $\theta_{A Q}, \theta_{Q B}$ and $\theta_{A B}$ ) are contained in the variancecovariance matrix. Assume that markers $A$ and $B$ are in LD with the trait locus $Q$, i.e., $D_{A Q} \neq 0, D_{Q B} \neq 0$. We may simultaneously test LD of marker $A$ and marker $B$ with trait locus $Q$, the gene substitution and dominant effects by testing $\alpha_{A}=\alpha_{B}=\delta_{A}=\delta_{B}=0$. From equation (3), we may test LD of markers $A$ and $B$ with the trait locus $Q$ and the gene substitution effect $\alpha_{Q}$ by testing $\alpha_{A}=\alpha_{B}=0$. From equation (4), we may test LD of markers $A$ and $B$ with the trait locus $Q$ and the dominant effect by testing $\delta_{A}=\delta_{B}=0$.

To test linkage, one may use the likelihood ratio test of the $\log$-likelihood $L$. Under the null hypothesis of no linkage between the major trait locus $Q$ and the markers, $\theta_{A Q}=$ $\theta_{Q B}=1 / 2$. Under the alternative hypothesis of linkage, $\theta_{A Q} \neq 1 / 2$ or $\theta_{Q B} \neq 1 / 2$. By comparing the difference of maximum log-likelihoods under the alternative and null hypotheses, we may use $\chi^{2}$ statistic to test the linkage. We will derive analytical formulas to explore the linkage interval mapping by the nuclear families in a similar way to Sham et $a l^{9}$ according to statistical theory of likelihood ratio tests. ${ }^{20}$

\section{Non-centrality parameters of association study}

Assume that there are no covariates. Then $\mu=\left(\beta, \alpha_{A}, \alpha_{B}, \delta_{A}, \delta_{B}\right)^{\tau}$. Consider the overall $\log$-likelihood $L=\sum_{i=1}^{I} L_{i}$, where $L_{i}$ is the log-likelihood of trait values $\mathrm{y}_{i}$ of the $i$-th family or individual. Let $\Sigma_{i}$ be the variance-covariance matrix of $\mathrm{y}_{i}$, and $X_{i}$ be its model matrix. Denote the total trait values $\mathrm{y}=\left(\mathrm{y}_{1}^{\tau}, \cdots, \mathrm{y}_{1}^{\hat{\tau}}\right)^{\tau}$, the total variance-covariance matrix by $\Sigma=\operatorname{diag}\left(\Sigma_{1}, \cdots, \Sigma_{I}\right)$, and the model matrix $X=\left(X_{1}^{\tau}, \cdots, X_{I}^{\tau}\right)^{\tau}$. Let $\beta, \hat{\alpha}_{A}, \hat{\alpha}_{B}, \hat{\delta}_{A}, \hat{\delta}_{B}, \hat{\Sigma}_{i}, \hat{\Sigma}$ be the maximum likelihood estimators of $\beta, \alpha_{A}, \alpha_{B}, \delta_{A}, \delta_{B}, \Sigma_{i}, \Sigma$. The estimate of $\mu$ is $\hat{\mu}=$ $\left[X^{\tau} \hat{\Sigma}{ }^{1} X\right]{ }^{1} X^{\tau} \hat{\Sigma}{ }^{1} \vec{y}=\left[\sum_{i=1}^{I} X_{i}^{\tau} \hat{\Sigma}_{i}{ }^{1} X_{i}\right]{ }^{1} \sum_{i=1}^{I} X_{i}^{\tau} \hat{\Sigma}_{i}{ }^{1} \vec{y}_{i}$. Let $H$ be a $q \times 5$ test matrix of rank $q$. Suppose that the total number of individuals is $N$. By Graybill, ${ }^{21}$ Chapter 6 , the test statistic of a hypothesis $H \mu=0$ is non-central $F(q, N-5)$ defined by

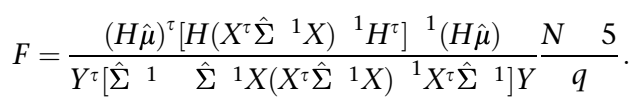

The non-centrality parameter of the test statistic $F$ can be calculated by $\lambda=(H \mu)^{\tau}\left[H\left[X^{\tau} \Sigma{ }^{1} X\right]{ }^{1} H^{\tau}\right]{ }^{1} H \mu$. If the data are composed of $n$ individuals of a population, Fan and Xiong ${ }^{14}$ worked out the non-centrality parameters to test if there are allele substitution and/or dominant effects and LDs between the markers and the major gene locus. In the following, we discuss a situation that the data are composed of both individual population data and family data.

Suppose that there are $n$ individuals of a population, and $n$ is sufficiently large. For each $y_{i}$ of the $n$ individuals, $\Sigma_{i}=\sigma^{2}$ and $X_{i}=\left(\begin{array}{llll}1 & x_{A i} & x_{B i} & Z_{A i} \\ Z_{B i}\end{array}\right), i=1,2, \cdots, n$. From formulas in Fan and Xiong, ${ }^{14}$ Appendix A and Appendix B, we may show that

$$
\frac{1}{n} \sum_{i=1}^{n} X_{i}^{\tau} \Sigma_{i}{ }^{1} X_{i}=\frac{1}{n \sigma^{2}} \sum_{i=1}^{n} X_{i}^{\tau} X_{i} \approx \frac{1}{\sigma^{2}} \operatorname{diag}\left(1, V_{A}, V_{D}\right),
$$

where $V_{A}$ and $V_{D}$ are additive and dominant variance-covariance matrices given in (2).

Secondly, suppose that there are $m$ trio families, and $m$ is sufficiently large. A trio family is composed of both parents and a single child. Notice that the means of $x_{A i}, x_{B i}, z_{A i}$ and $Z_{B i}$ are 0 . Let $K_{f}=\left(x_{A f} x_{B f} Z_{A f} Z_{B f}\right)$ and $K_{m}=\left(x_{A m} x_{B m} Z_{A m} Z_{B m}\right)$. We show in Appendix $A$ that the covariance matrix between parents and their offspring is

$$
\mathrm{E} K_{f}^{\tau} K_{1}=\mathrm{E} K_{m}^{\tau} K_{1}=\left(\begin{array}{cc}
V_{A} / 2 & O_{2} \\
O_{2} & O_{2}
\end{array}\right)
$$

where $K_{1}=\left(\begin{array}{lllll}x_{A 1} & x_{B 1} & z_{A 1} & z_{B 1}\end{array}\right)$ and $O_{2}$ is zero $2 \times 2$ matrix. For each of the trio families, the variance-covariance $\Sigma_{i}$ is a $3 \times 3$ matrix whose inverse is

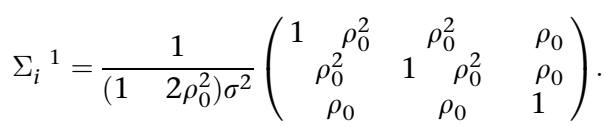

Using equations (5), (6), and (7), we show in Appendix B

$$
\begin{aligned}
& \frac{1}{m} \sum_{i=n+1}^{n+m} X_{i}^{\tau} \Sigma_{i}{ }^{1} X_{i} \approx \frac{1}{\left(1 \quad 2 \rho_{0}^{2}\right) \sigma^{2}} \\
& \left(\begin{array}{ccccc}
3 & 4 \rho_{0} & \multicolumn{2}{c}{0} & 0 \\
0 & (3 & 2 \rho_{0} & \left.2 \rho_{0}^{2}\right) V_{A} & \\
0 & \multicolumn{2}{c}{0} & (3 & \left.2 \rho_{0}^{2}\right) V_{D}
\end{array}\right) .
\end{aligned}
$$

Thirdly, suppose that there are $k$ nuclear families each of them has both parents and two offspring, and the correlation of the two offspring is $\rho_{12}$. Assume that $k$ is sufficiently large. For each family, the variance-covariance $\Sigma_{i}$ is a $4 \times 4$ matrix whose inverse is

$$
\begin{aligned}
& \Sigma_{i}{ }^{1}=\frac{1}{\sigma^{2}}
\end{aligned}
$$

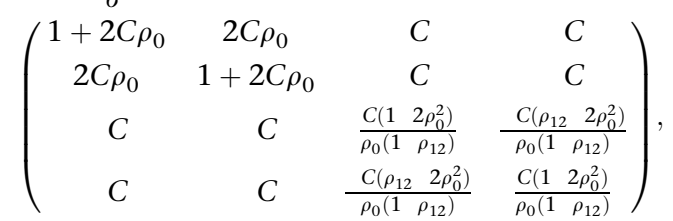

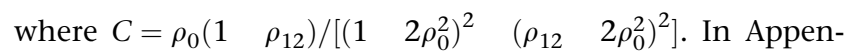
dix $C$, we show that the covariance matrix between two offspring is 


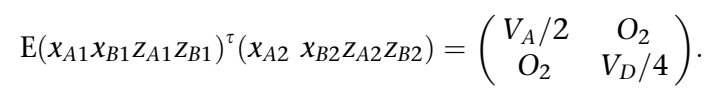

Using equations (5), (6), (9) and (10), we show in Appendix $\mathrm{D}$ that

$$
\frac{1}{k} \sum_{i=n+m+1}^{n+m+k} X_{i}^{\tau} \Sigma_{i}^{1} X_{i} \approx \operatorname{diag}\left(d_{11}, d_{22} V_{A}, d_{44} V_{D}\right) / \sigma^{2}
$$

where the constants are given by $d_{11}=2\left[1+4 C \rho_{0}\right.$ $\left.4 C+C / \rho_{0}\right], d_{22}=2+4 C\left(\rho_{0} \quad 1\right)+C\left(\begin{array}{lll}2 & \rho_{12} & 2 \rho_{0}^{2}\end{array}\right) /\left[\rho_{0}(1\right.$ $\left.\left.\left.\rho_{12}\right)\right], d_{44}=2\left(1+2 C \rho_{0}\right)+C\left[\begin{array}{llll}4 & \left.2 \rho_{0}^{2}\right) & \left(\rho_{12}\right. & 2 \rho_{0}^{2}\end{array}\right)\right] /\left[2 \rho_{0}(1\right.$ $\left.\rho_{12}\right)$ ]. Combine the $n$ individuals, $m$ trio families, and $k$ families with two offspring. Define $a_{1}=n+m\left(\begin{array}{ll}1 & 2 \rho_{0}^{2}\end{array}\right)^{1}$ $\left(\begin{array}{ll}3 & 4 \rho_{0}\end{array}\right)+k d_{11}, a_{2}=n+m\left(1 \quad 2 \rho_{0}^{2}\right)^{1}\left(\begin{array}{lll}3 & 2 \rho_{0} & 2 \rho_{0}^{2}\end{array}\right)+$ $k d_{22}, a_{3}=n+m\left(\begin{array}{lll}1 & 2 \rho_{0}^{2}\end{array}\right)^{1}\left(\begin{array}{ll}3 & 2 \rho_{0}^{2}\end{array}\right)+k d_{44}$. Then equations (5), (8) and (11) lead to

$$
\sum_{i=1}^{n+m+k} X_{i}^{\tau} \Sigma_{i}{ }^{1} X_{i} \approx \operatorname{diag}\left(a_{1}, a_{2} V_{A}, a_{3} V_{D}\right) / \sigma^{2}
$$

To test if there are additive and dominant effects, we may test the hypothesis $H_{A B, a d}: \alpha_{A}=\alpha_{B}=\delta_{A}=\delta_{B}=0$. Then the test matrix $H$ is defined by

$$
H=\left(\begin{array}{lllll}
0 & 1 & 0 & 0 & 0 \\
0 & 0 & 1 & 0 & 0 \\
0 & 0 & 0 & 1 & 0 \\
0 & 0 & 0 & 0 & 1
\end{array}\right) .
$$

Let us denote the corresponding $F$-test statistic by $F_{A B, a d}$, and the non-centrality parameter by $\lambda_{A B, a d}$. Then we have from (3), (4), and (12) that

$$
\begin{aligned}
& \lambda_{A B, a d} \approx \frac{1}{\sigma^{2}}\left[a_{2}\left(\alpha_{A} \alpha_{B}\right) V_{A}\left(\begin{array}{c}
\alpha_{A} \\
\alpha_{B}
\end{array}\right)+a_{3}\left(\delta_{A} \delta_{B}\right) V_{D}\left(\begin{array}{c}
\delta_{A} \\
\delta_{B}
\end{array}\right)\right] \\
& =\frac{1}{\sigma^{2}}\left[2 a_{2} \alpha_{Q}^{2}\left[P_{b} P_{B} D_{A Q}^{2} \quad 2 D_{A Q} D_{A B} D_{Q B}+P_{a} P_{A} D_{Q B}^{2}\right] /\right.
\end{aligned}
$$

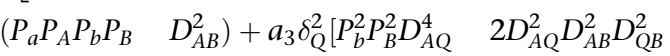

$$
\begin{aligned}
& \left.\left.+P_{a}^{2} P_{A}^{2} D_{\mathrm{QB}}^{4}\right] /\left(P_{a}^{2} P_{A}^{2} P_{b}^{2} P_{B}^{2} \quad D_{A B}^{4}\right)\right] \\
& =\frac{1}{\sigma^{2}}\left[a_{2} \sigma_{g a}^{2}\left[R_{A Q}^{2} \quad 2 R_{A Q} R_{A B} R_{Q B}+R_{Q B}^{2}\right]\left(\begin{array}{ll}
1 & R_{A B}^{2}
\end{array}\right)\right. \\
& \left.+a_{3} \sigma_{g d}^{2}\left[R_{A Q}^{4} \quad 2 R_{A Q}^{2} R_{A B}^{2} R_{Q B}^{2}+R_{Q B}^{4}\right] /\left(1 \quad R_{A B}^{4}\right)\right] .
\end{aligned}
$$

Assume that the two markers $A$ and $B$ are in linkage equilibrium, then $D_{A B}=0$. Moreover, assume that the trait locus $Q$ is in LD with marker $A$ but not with marker $B$, then $D_{\mathrm{Q} B}=0$ and $D_{A Q} \neq 0$. Then $\lambda_{A B, a d} \approx\left[1 / \sigma^{2}\right]\left[a_{2} \sigma_{g a}^{2} R_{A Q}^{2}+a_{3} \sigma_{g d}^{2} R_{A Q}^{4}\right]$, which only involves marker $A$ and can be written as $\lambda_{A, a d}$ Correspondingly, we denote the $F$-test statistic by $F_{A, a d}$. Similarly, $\lambda_{A, a} \approx\left[a_{2} / \sigma^{2}\right] \sigma_{g a}^{2} R_{A Q}^{2}$ is the non-centrality parameter of a test statistic $F_{A, a}$. To test the other hypotheses, we may get the non-centrality parameters in a similar way by taking appropriate test matrices $H$. To test if there is dominant effect, we may test the hypothesis $H_{A B, d}: \delta_{A}=\delta_{B}=0$. The non-centrality parameter is $\lambda_{A B, d} \approx \frac{a_{3}}{\sigma^{2}} \sigma_{g d}^{2}\left[R_{A Q}^{4}\right.$ $\left.2 R_{A Q}^{2} R_{A B}^{2} R_{O B}^{2}+R_{O B}^{4}\right] /\left(1 \quad R_{A B}^{4}\right)$. To test if there is an additive or substitution effect, we may test the hypothesis $H_{A B, a}: \alpha_{A}=\alpha_{B}=0$. The non-centrality parameter is $\lambda_{A, B a} \approx$ $\frac{a_{2}}{\sigma^{2}} \sigma_{g a}^{2}\left[R_{A Q}^{2} \quad 2 R_{A Q} R_{A B} R_{Q B}+R_{Q B}^{2}\right] /\left(1 \quad R_{A B}^{2}\right)$. The corresponding $F$-test statistic is denoted by $F_{A B, a}$.

\section{Non-centrality parameters of linkage studies}

Consider a nuclear family with $k$ children and both parents. Under the null hypothesis of no linkage between the trait locus and markers, the correlation of each sib-pair is

$$
\rho_{N}=\frac{\sigma_{g a}^{2}}{2 \sigma^{2}}+\frac{\sigma_{g d}^{2}}{4 \sigma^{2}}+\frac{\sigma_{G a}^{2}}{2 \sigma^{2}}+\frac{\sigma_{G d}^{2}}{4 \sigma^{2}} .
$$

The expected log-likelihood is $\mathrm{E}\left(2 L_{\text {Null }}\right)=(k+2)$

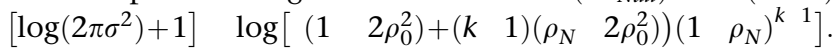
Under the alternative hypothesis of linkage between the trait locus and marker $A$, the correlation between a sib-pair is $C_{i}=\operatorname{Cov}\left(y_{1}, y_{2} \mid \pi_{A}=i / 2\right) / \sigma^{2}=\left(\sigma_{g a}^{2}+\sigma_{g d}^{2}\right) P\left(\pi_{Q}=1 \mid \pi_{A}=i / 2\right) /$ $\sigma^{2}+\frac{\sigma_{g a}^{2}}{2} P\left(\pi_{Q}=1 / 2 \mid \pi_{A}=i / 2\right) / \sigma^{2}+\left[\sigma_{G a}^{2} / 2+\sigma_{G d}^{2} / 4\right] / \sigma^{2}, i=0,1,2$. From Haseman and Elston, ${ }^{22}$ Table IV, we have

$$
\begin{aligned}
& C_{2}=\left[\left(\sigma_{g a}^{2}+\sigma_{g d}^{2}\right) \psi_{A}^{2}+\sigma_{g a}^{2} \psi_{A}\left(1 \quad \psi_{A}\right)+\sigma_{G a}^{2} / 2+\sigma_{G d}^{2} / 4\right] / \sigma^{2} \\
& C_{1}=\left[\left(\sigma_{g a}^{2}+\sigma_{g d}^{2}\right) \psi_{A}\left(1 \quad \psi_{A}\right)+\sigma_{g a}^{2}\left[\begin{array}{lll}
1 & 2 \psi_{A}(1 & \psi_{A}
\end{array}\right)\right] / 2 \\
& \left.+\sigma_{G a}^{2} / 2+\sigma_{G d}^{2} / 4\right] / \sigma^{2} \\
& C_{0}=\left[\left(\sigma_{g a}^{2}+\sigma_{g d}^{2}\right)\left(1 \quad \psi_{A}\right)^{2}+\sigma_{g a}^{2} \psi_{A}\left(1 \quad \psi_{A}\right)+\sigma_{G a}^{2} / 2+\sigma_{G d}^{2} / 4\right] / \sigma^{2} .
\end{aligned}
$$

The expected log-likelihood under the alternative hypothesis of linkage is

$$
\begin{aligned}
& \mathrm{E}\left(2 L_{\text {random }, A}\right)=(k+2)\left[\log \left(2 \pi \sigma^{2}\right)+1\right] \\
& \sum_{\pi_{12 A}} \cdots \sum_{\pi_{k} 1, k A} P\left(\pi_{12 A}\right) \cdots P\left(\pi_{k} 1, k A\right) \text {. } \\
& \log \operatorname{det}\left(\begin{array}{cccccc}
1 & 0 & \rho_{0} & \rho_{0} & \cdots & \rho_{0} \\
0 & 1 & \rho_{0} & \rho_{0} & \cdots & \rho_{0} \\
\rho_{0} & \rho_{0} & 1 & C_{2 \pi_{12 A}} & \cdots & C_{2 \pi_{1 k A}} \\
\rho_{0} & \rho_{0} & C_{2 \pi_{21 A}} & 1 & \cdots & C_{2 \pi_{2 k A}} \\
\vdots & \vdots & \vdots & \vdots & \cdots & \vdots \\
\rho_{0} & \rho_{0} & C_{2 \pi_{k 1}} & C_{2 \pi_{k 2 A}} & \cdots & 1
\end{array}\right) \text {, }
\end{aligned}
$$

where $P\left(\pi_{i j A}=0\right)=P\left(\pi_{i j A}=1\right)=1 / 4$ and $P\left(\pi_{i j A}=1 / 2\right)=1 / 2$. From Stuart and $\mathrm{Ord}^{20}$ the non-centrality parameter for linkage of the nuclear family is equal to $\lambda_{\text {linkage } A}=\mathrm{E}\left(2 L_{\text {random }, A}\right)-$ $\mathrm{E}\left(2 L_{N \text { Null }}\right)$. If $k=2$, it can be shown that $\lambda_{\text {linkage, } A}=$ $\log \left[1 \quad 4 \rho_{0}^{2} \quad \rho_{N}^{2}+4 \rho_{0}^{2} \rho_{N}\right] \quad \sum_{i=0}^{2} P\left(\pi_{12 A}=i / 2\right) \log \left[1 \quad 4 \rho_{0}^{2}\right.$ $\left.C_{i}^{2}+4 \rho_{0}^{2} C_{i}\right]$. 
Under the alternative hypothesis of linkage between the trait locus and markers $A$ and $B$, the correlation between a sib-pair is given by for $i, j=0,1,2$

$$
\begin{aligned}
C_{i j}= & \operatorname{Cov}\left(y_{1}, y_{2} \mid \pi_{12 A}=i / 2, \pi_{12 B}=j / 2\right) / \sigma^{2} \\
= & {\left[\left(\sigma_{g a}^{2}+\sigma_{g d}^{2}\right) P\left(\pi_{12 Q}=1 \mid \pi_{12 A}=i / 2, \pi_{12 B}=j / 2\right)\right.} \\
& +\frac{\sigma_{g a}^{2}}{2} P\left(\pi_{12 Q}=1 / 2 \mid \pi_{12 A}=i / 2, \pi_{12 B}=j / 2\right) \\
& \left.+\sigma_{G a}^{2} / 2+\sigma_{G d}^{2} / 4\right] / \sigma^{2} .
\end{aligned}
$$

To calculate $C_{i j}$, we need to calculate the joint distribution of $\pi_{12 A}, \pi_{12 Q}$ and $\pi_{12 B}$ of a sib-pair under the alternative hypothesis of linkage. Assume that there is no interference for disjoint regions of the chromosome. Then we have

$$
\begin{aligned}
& P\left(\pi_{12 A}=i_{A}, \pi_{12 Q}=i_{Q}, \pi_{12 B}=i_{B}\right) \\
& =P\left(\pi_{12 A}=i_{A}, \pi_{12 Q}=i_{Q}\right) P\left(\pi_{12 B}=i_{B} \mid \pi_{12 A}=i_{A}, \pi_{12 Q}=i_{Q}\right) \\
& =P\left(\pi_{12 A}=i_{A} \mid \pi_{12 Q}=i_{Q}\right) P\left(\pi_{12 Q}=i_{Q}\right) P\left(\pi_{12 B}=i_{B} \mid \pi_{12 Q}=i_{Q}\right) .
\end{aligned}
$$

From Haseman and Elston, ${ }^{22}$ Table IV, we may construct the joint distribution of $\pi_{12 Q}, \pi_{12 A}$ and $\pi_{12 \mathrm{~B}}$ by relation (15), and the results are presented in Table 3 of Fan. ${ }^{17}$ Based on the results, we can calculate $C_{i j}, i, j=0,1,2$, which are given in Appendix D of Fan. ${ }^{17}$ The expected log-likelihood under the alternative hypothesis of linkage is $\mathrm{E}\left(2 L_{\text {random }, A B}\right)=-(k+2)\left[\log \left(2 \pi \sigma^{2}\right)+1\right]-\Sigma_{\pi_{12 A}} \quad \Sigma_{\pi_{12 B}} \cdots \quad \Sigma_{\pi_{k-1, k A}}$ $\Sigma_{\pi_{k-1, k B}} P\left(\pi_{12 A}\right) P\left(\pi_{12 B}\right) \cdots P\left(\pi_{k-1, k A}\right) P\left(\pi_{k-1, k B}\right)$

$$
\log \operatorname{det}\left(\begin{array}{cccccc}
1 & 0 & \rho_{0} & \rho_{0} & \cdots & \rho_{0} \\
0 & 1 & \rho_{0} & \rho_{0} & \cdots & \rho_{0} \\
\rho_{0} & \rho_{0} & 1 & C_{2 \pi_{12 A}, 2 \pi_{12 B}} & \cdots & C_{2 \pi_{1 k A}, 2 \pi_{1 k B}} \\
\rho_{0} & \rho_{0} & C_{2 \pi_{21 A}, 2 \pi_{21 B}} & 1 & \cdots & C_{2 \pi_{2 k A}, 2 \pi_{2 k B}} \\
\vdots & \vdots & \vdots & \vdots & \cdots & \vdots \\
\rho_{0} & \rho_{0} & C_{2 \pi_{k 1 A}, 2 \pi_{k 1 B}} & C_{2 \pi_{k 2 A}, 2 \pi_{k 2 B}} & \cdots & 1
\end{array}\right) \text {, }
$$

where $P\left(\pi_{i j B}=0\right)=P\left(\pi_{i j B}=1\right)=1 / 4$ and $P\left(\pi_{i j B}=1 / 2\right)=1 / 2$ such as those for marker $A$. From Stuart and Ord $^{20}$, the non-centrality parameter for linkage of the nuclear family is equal to $\lambda_{\text {linkage, } A B}=\mathrm{E}\left(2 L_{\text {random }, A B}\right)-\mathrm{E}\left(2 L_{\text {Null }}\right)$. If $k=2$, it can be shown that $\quad \lambda_{\text {linkage }, A B}=\log \left[\begin{array}{lll}1 & 4 \rho_{0}^{2} & \rho_{N}^{2}+4 \rho_{0}^{2} \rho_{N}\end{array}\right] \quad \sum_{i, j=0}^{2} P\left(\pi_{12 A}=\right.$ $i / 2) P\left(\pi_{12 B}=j / 2\right) \log \left[1 \quad 4 \rho_{0}^{2} \quad C_{i j}^{2}+4 \rho_{0}^{2} C_{i j}\right]$.

\section{Power calculation and comparison}

Let us denote heritability by $h^{2}$ which is defined by $h^{2}=\sigma_{g a}^{2} / \sigma^{2}$. In the power calculations, we take the additive polygenic variance $\sigma_{G a}^{2}=0.10$, polygenic dominant variance $\sigma_{G d}^{2}=0.05$, the equal allele frequencies $P_{A}=q_{1}=P_{B}=0.5$ at the two markers $A$ and $B$, and the QTL $Q$. Moreover, suppose that $\mu_{11}=a, \mu_{12}=\mu_{21}=d$ and $\mu_{22}=-a$.

Suppose that the map distance $\lambda_{A B}$ between marker $A$ and marker $B$ is known. Under the assumption of no interference, we may calculate the recombination fraction by
Haldane's map function $\theta_{A B}=\left[1-\exp \left(-2 \lambda_{A B}\right)\right] / 2$. Similarly, we may calculate the recombination fractions $\theta_{A Q}$ and $\theta_{\mathrm{Q} B}$ by the map distances $\lambda_{A Q}$ and $\lambda_{Q B}$. Assume that marker $A$ and marker $B$ are in linkage equilibrium, i.e., $D_{A B}=0$, the genetic distances $\lambda_{A B}=5 \mathrm{cM}, \lambda_{A Q}=\lambda_{Q B}=2.5 \mathrm{cM}$, and the heritability $h^{2}=0.25$. Suppose we have a sample with $n=100$ individuals, $m=30$ trio families, and $k=20$ nuclear families with two offspring. Assume that the IBD proportions shared by the two offspring in the $k=20$ families at both markers $A$ and $B$ are $\pi_{A}=\pi_{B}=0.5$, and the probability of sharing two alleles IBD at markers $A$ and $B$ are $\Delta_{A}=\Delta_{B}=0.5$. Figure 1 shows the power of the test statistics $F_{A B, a d}, F_{A B, a}, F_{A, a d}$, and $F_{A, a}$ against the disequilibrium coefficient $D_{A Q}$ when $D_{Q B}=0.15$ for a mode of dominant inheritance with $a=d=1.0$, and a mode of recessive inheritance with $a=1.0$, $d=-0.5$, respectively. Several features are interesting in the two graphs of Figure 1 . First, the power of $F_{A B, a d}$ and $F_{A B, a}$ are higher than that of $F_{A, a d}$ and $F_{A, a}$. Hence, the regression mapping which uses two markers $A$ and $B$ has its advantage
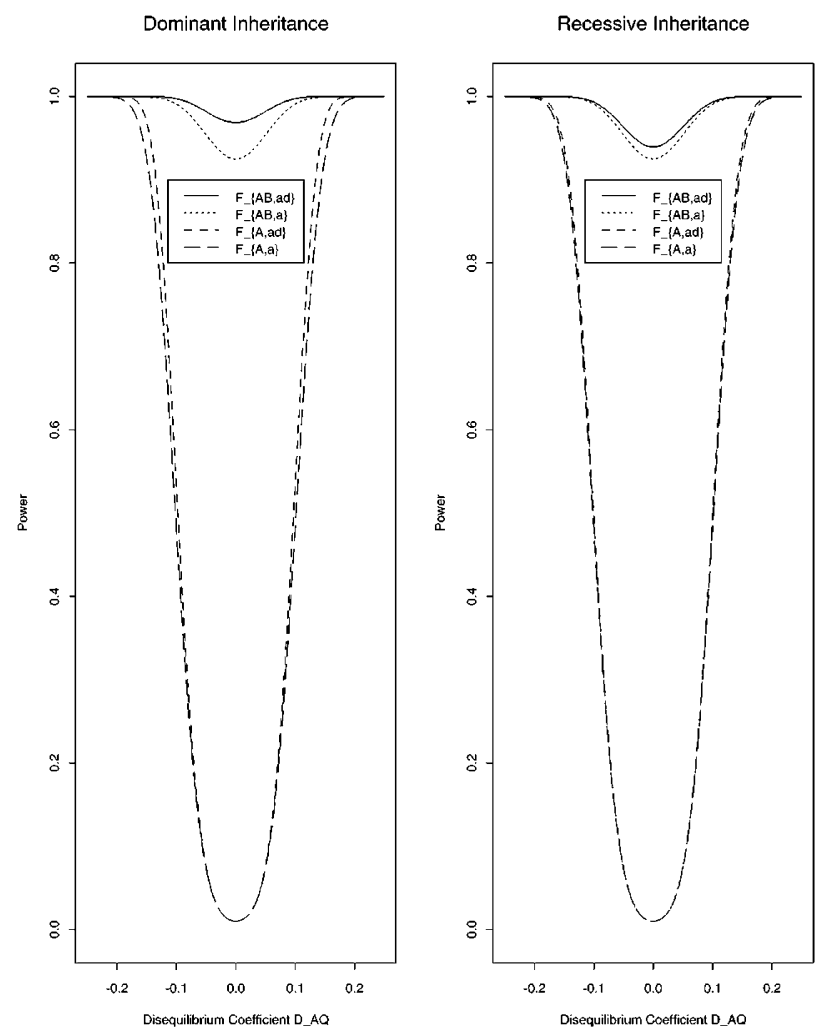

Figure 1 Power of test statistics $F_{A B, a d}, F_{A B, a}, F_{A, a d}$, and $F_{A, a}$ against disequilibrium coefficient $D_{A Q}$ at 0.01 significant level, when $q_{1}=P_{A}=P_{B}=0.50, D_{A B}=0.0, D_{\mathrm{QB}}=0.15, h^{2}=0.25, n=100$, $m=30, k=20, \pi_{A}=\pi_{B}=\Delta_{A}=\Delta_{B}=0.5, \lambda_{A B}=5 \mathrm{cM}, \lambda_{A Q}=\lambda_{\mathrm{Q} B}=2.5 \mathrm{cM}$, $\sigma_{G a}^{2}=0.10, \sigma_{G d}^{2}=0.05$ for a mode of dominant inheritance $a=d=1.0$, and a mode of recessive inheritance $a=1.0, d=-0.5$, respectively. 
over the one marker mapping which only uses one marker $A$ or $B$. Second, the statistic $F_{A B, a d}$ has higher power than that of $F_{A B, a}$, and the statistic $F_{A, a d}$ has higher power than that of $F_{A, a}$. Thus, excluding the dominant variance from the analysis when it does exist would lose power. Third, as expected, when $D_{A Q}=0$ the power to detect LD using only marker $A$ is minimal. More interestingly, when $D_{A Q}=0.15$ the power is still higher using the flanking two markers than using only marker $A$.

Figure 2 shows the power of the test statistics $F_{A B, a d}, F_{A B, a}$, $F_{A, a d}$, and $F_{A, a}$ against the heritability $h^{2}$ when $D_{A B}=0.10$ and $D_{A Q}=D_{Q B}=0.15$ for a mode of dominant inheritance with $a=d=1.0$, and a mode of recessive inheritance with $a=1.0$, $d=-0.5$, respectively. The other parameters are the same as those of Figure 1. Among the features observed in Figure 1 , the power is reasonably high when the heritability $h^{2}$ is bigger than 0.15 . To compare the power of population based and family based methods, Figure 3 shows the power of the test statistics $F_{A B, a d}$ and $F_{A B, a}$ for a mode of dominant inheritance with $a=d=1.0$, and a mode of recessive inheri-
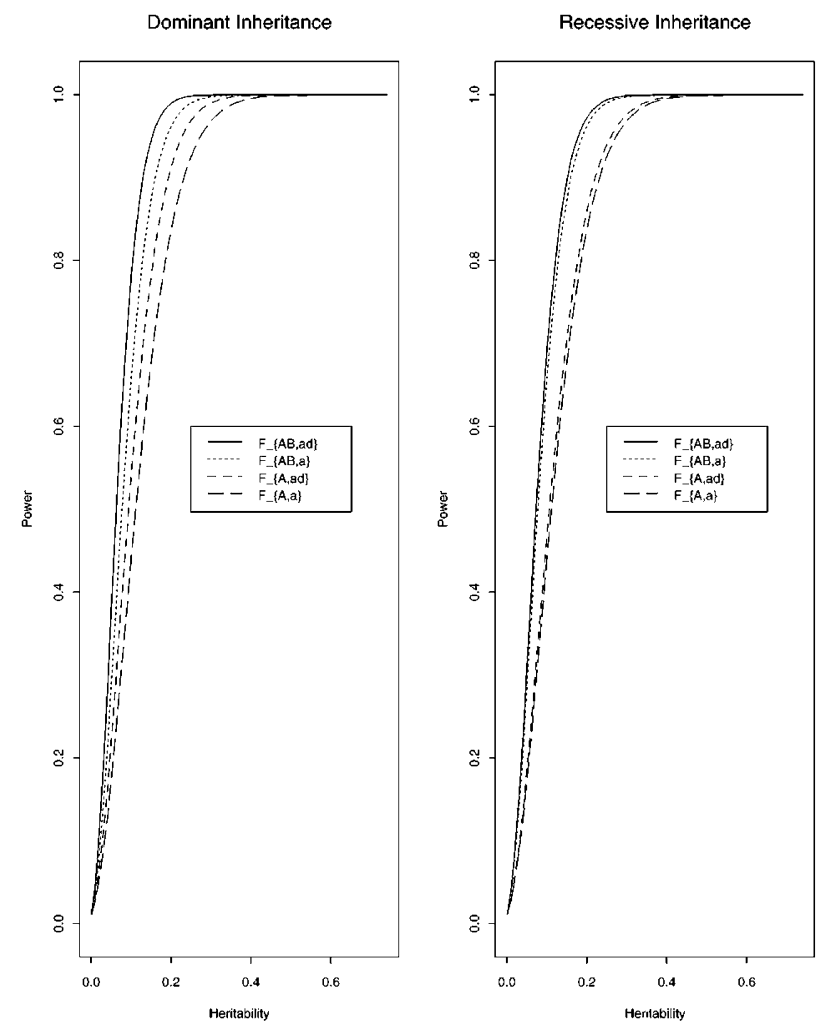

Figure 2 Power of test statistics $F_{A B, a d,} F_{A B, a}, F_{A, a d}$, and $F_{A, a}$ against heritability $h^{2}$ at 0.01 significant level, when $q_{1}=P_{A}=P_{B}=$ $0.50, D_{A B}=0.10, D_{A Q}=D_{Q B}=0.15, n=100, m=30, k=20, \pi_{A}=\pi_{B}=$ $\Delta_{A}=\Delta_{B}=0.5, \lambda_{A B}=5 \mathrm{cM}, \lambda_{A Q}=\lambda_{Q B}=2.5 \mathrm{cM}, \sigma_{C a}^{2}=0.10, \sigma_{G d}^{2}=0.05$ for a mode of dominant inheritance $a=d=1.0$, and a mode of recessive inheritance $a=1.0, d=-0.5$, respectively. tance with $a=1.0, d=-0.5$, respectively. For Figure 3, population data contain $n=252$ individuals, but no family data $(m=k=0)$. For dominant inheritance of Figure 3 , the data contain $m=84$ trio families $(n=k=0)$. For recessive inheritance of Figure 3 , the data contain $k=63$ nuclear families each has two offspring $(n=m=0)$. Notice that $m=84$ or $k=63$ family data contain 252 individuals, and thus the number of individuals is the same as that of the population data. We can see that population based method is more powerful than the family based method for the same number of individuals.

In a population, the $\mathrm{LD}$ can exist due to mutations at the trait locus. In the absence of tight linkage between the trait locus and a marker, the recombination between the marker locus and the trait locus can rapidly dissipate the disequilibrium from generation to generation. Denote the frequency of haplotype $A Q$ at the generation when the mutations occur by $P(A Q)(0)$. Then LD coefficient is $D_{A Q}(0)=$ $P(A Q)(0)-q_{1} P_{A}$ for the generation when the mutations occur. For the following generations, the disequilibrium
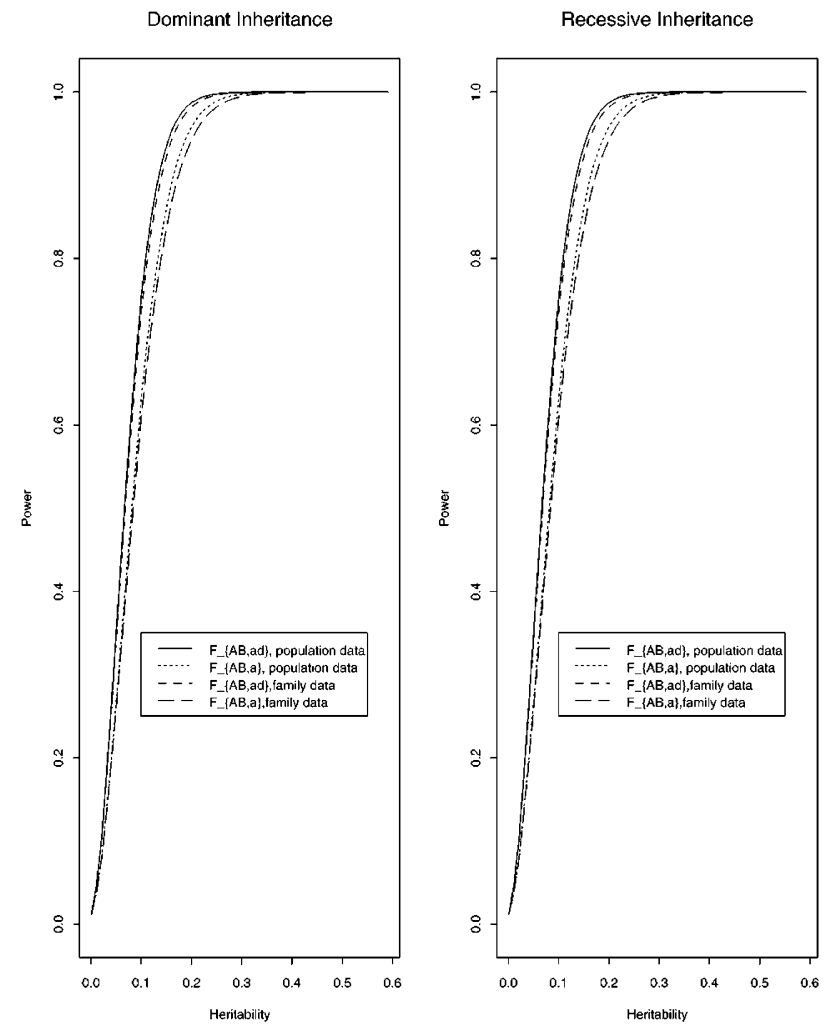

Figure 3 Power of test statistics $F_{A B, a d}$ and $F_{A B, a}$ against heritability $h^{2}$ at 0.01 significant level for a mode of dominant inheritance $a=d=1.0$, and a mode of recessive inheritance $a=1.0$, $d=-0.5$, respectively. For population data $n=252, m=k=0$; for dominant family data $n=k=0, m=84$; for recessive family data $n=m=0, k=63$. Other parameters are the same as those of Figure 2. 
coefficient is reduced by a factor $1-\theta_{A Q}$ in each generation. ${ }^{23}$ Suppose that the mutation is already $T$ generation old. Then the LD coefficient is $D_{A Q}(T)=D_{A Q}(0)\left(1-\theta_{A Q}\right)^{T}$. Similarly, the other LD coefficients are $D_{A B}(T)=D_{A B}(0)(1-$ $\left.\theta_{A B}\right)^{T}$ and $D_{Q B}(T)=D_{Q B}(0)\left(1-\theta_{Q B}\right)^{\mathrm{T}}$.

Assume that the map distance between marker $A$ and marker $B$ is $\lambda_{A B}=5 \mathrm{cM}$, and the other parameters are given by $D_{A B}(0)=0.20, D_{A Q}(0)=D_{Q B}(0)=0.25, \quad h^{2}=0.25, \lambda_{A B}=5 \mathrm{cM}$, $n=100, m=30, k=20, T=30, \pi_{A}=\pi_{B}=0.5, \Delta_{A}=\Delta_{B}=0.25$. Figure 4 shows the power of the test statistics $F_{A B, a d}, F_{A B . a}, F_{A . a d}$, and $F_{A, a}$ against the recombination fraction $\theta_{A Q}$ for a mode of dominant inheritance with $a=d=1.0$, and a mode of recessive inheritance with $a=1.0, d=-0.5$, respectively. We can see that the power curves of $F_{A B, a d}$ and $F_{A B, a}$ are very high, although the power curves of $F_{A . a d}$ and $F_{A, a}$ decrease very rapidly as the recombination fraction $\theta_{A Q}$ increases. Hence, high resolution $\mathrm{LD}$ mappings have advantage to do fine gene mappings, and appropriate for the dense marker maps such as single nucleotide polymorphisms on human genome. To investigate the effect of the age of the mutation
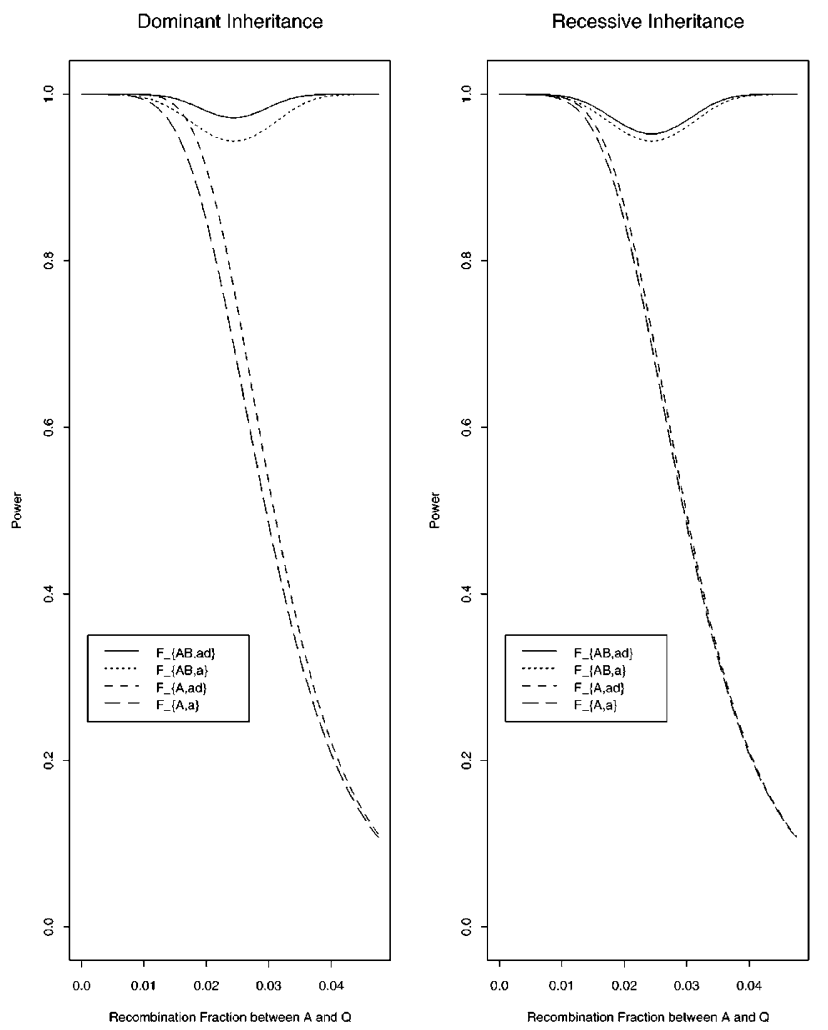

Figure 4 Power curves of the test statistics $F_{A B, a d}, F_{A B, a}, F_{A, a d}$ and $F_{A, a}$ against the recombination fraction $\theta_{A Q}$ at 0.01 significant level, when $q_{1}=P_{A}=P_{B}=0.50, D_{A B}(0)=0.20, D_{A Q}(0)=D_{Q B}(0)=$ $0.25, h^{2}=0.25, \lambda_{A B}=5 \mathrm{cM}, T=30, n=100, m=30, k=20, \pi_{A}=\pi_{B}=0.5$, $\Delta_{A}=\Delta_{B}=0.25, \sigma_{G a}^{2}=0.10, \sigma_{G d}^{2}=0.05$ for a mode of dominant inheritance $a=d=1.0$, and a mode of recessive inheritance $a=1.0$, $d=-0.5$, respectively. on the power, Figure 5 shows the power curves against the position of markers. In the Figure, the QTL locates at $15 \mathrm{~cm}$ which is flanked by two markers $A$ and $B$. One marker is one the right-hand side of the QTL, and the other is on the left-hand side with equal distance to the QTL. The power decreases quickly when the age of the mutation increases. For a mutation which is 30 generations old, one should expect very low power if the markers locate $5 \mathrm{~cm}$ away from the QTL.

To explore the linkage interval mapping, we take a sample of $k=250$ nuclear families each has two offspring. Multiplying $\lambda_{\text {linkage, } A}$ and $\lambda_{\text {linkage, } A B}$ by $k$, we may calculate the non-centrality parameters for the linkage mapping using marker $A$ and the linkage interval mapping using markers $A$ and $B$. Moreover, assume that the genetic distances are $\lambda_{A B}=30 \mathrm{cM}$, and $\lambda_{A Q}=\lambda_{Q B}=15 \mathrm{cM}$, i.e., the QTL $Q$ is right in the middle between markers $A$ and $B$. Figure 6 gives power curves of linkage interval mapping by markers $A$ and $B$, and linkage mapping by marker $A$ against heritability $h^{2}$ for a mode of dominant inheritance with
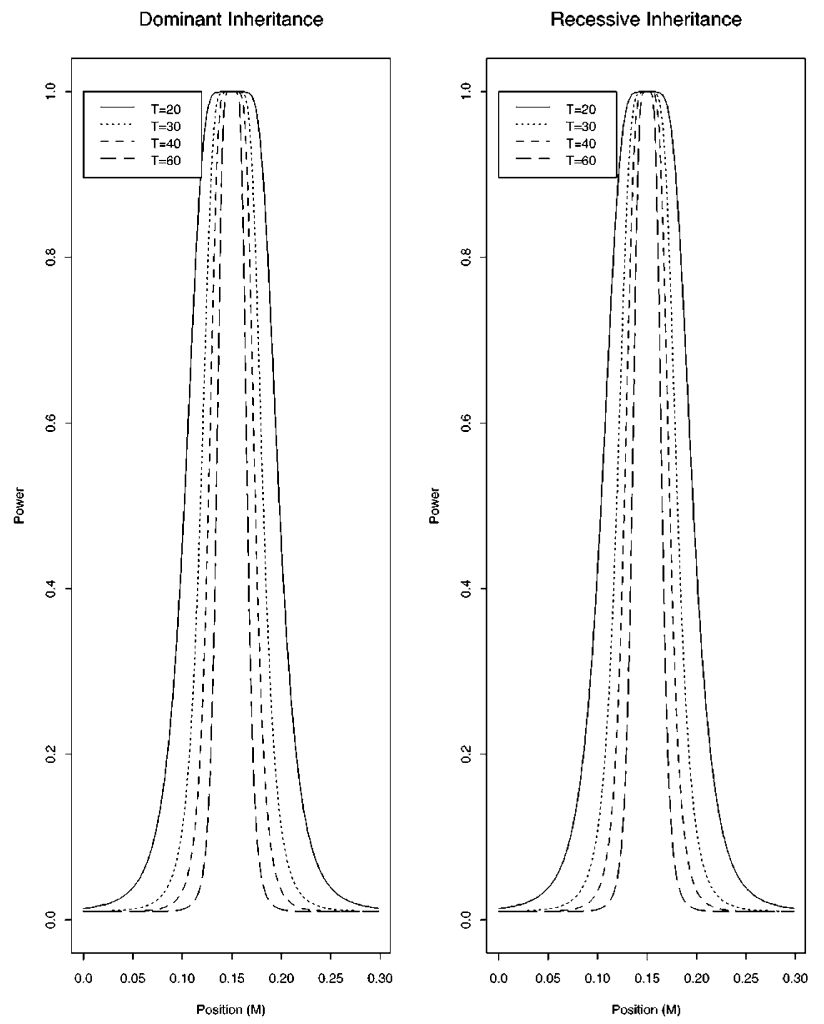

Figure 5 Power curves of the test statistics $F_{A B, a d}$ against the position of markers at 0.01 significant level for a mode of dominant inheritance $a=d=1.0$, and a mode of recessive inheritance $a=1.0, d=-0.5$, respectively. The QTL locates at $15 \mathrm{~cm}$ which is flanked by two markers $A$ and $B$. Here the mutation age $T=20,30,40,60$, and the other parameters are the same as those in Figure 4. 

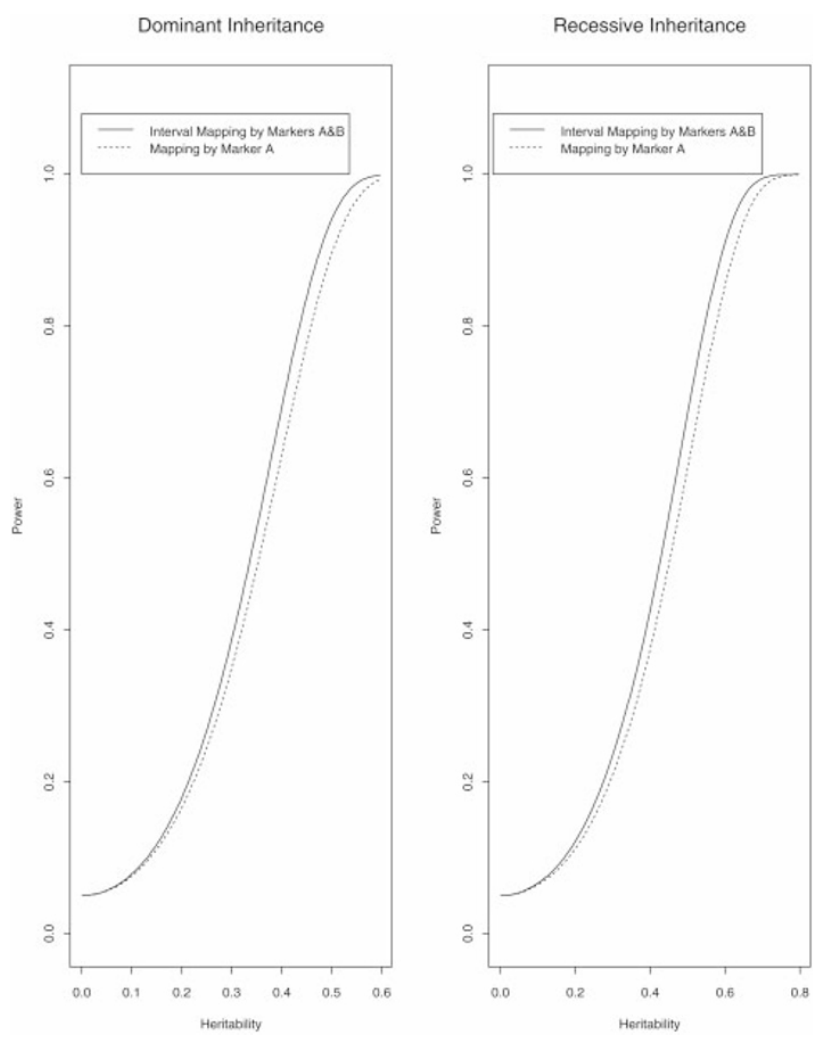

Figure 6 Power curves of the linkage interval mapping by markers $A$ and $B$, and linkage mapping by marker $A$ against the heritability $h^{2}$, when $q_{1}=P_{A}=P_{B}=0.50, \lambda_{A B}=30 \mathrm{cM}, \lambda_{A Q}=\lambda_{Q B}=$ $15 \mathrm{CM}, k=250, \sigma_{G a}^{2}=0.10, \sigma_{G d}^{2}=0.05$, at 0.05 significant level for a mode of dominant inheritance $a=d=1.0$, and a mode of recessive inheritance $a=1.0, d=-0.5$, respectively.

$a=d=1.0$, and a mode of recessive inheritance with $a=1.0$, $d=-0.5$, respectively. It is clear that the power of interval linkage mapping using both markers $A$ and $B$ is higher than that of linkage mapping using only one marker $A$.

\section{Discussion}

In this paper, we investigate variance component models of both high resolution LD mapping and linkage analysis for QTL. The models are based on family pedigree and population data. We consider likelihoods which utilizes flanking marker information. The likelihoods jointly include recombination fractions, LD coefficients, the average allele substitution effect and allele dominant effect as parameters. The linkage parameters are contained in the variance-covariance matrix. The parameters of $\mathrm{LD}$ and gene effects are contained in the regression coefficients. ${ }^{8,9,11,12}$ The model simultaneously takes care of the linkage, LD and the effects of the putative trait locus $Q$, and hence clearly demonstrates that linkage analysis and LD mapping are complimentary, not exclusive, methods for QTL mapping. The family data which have at least two offspring contain information for both linkage and association, and population data and trio family data which have two parents and only one offspring contain information for association. By combining the family and population data in the analysis, one may expect to get better results than that by analysing them separately.

Linkage analysis can localize genetic trait loci in broad chromosome regions of a few $\mathrm{cm}(<10 \mathrm{~cm})$, and is less sensitive to population admixture than LD mapping. In practice, one may carry out linkage analysis as a first step to obtain prior suggestive linkage based on a sparse marker map. By performing linkage interval mappings, one may get higher power than that of using only one marker. With prior linkage in hand, LD mapping can be used to get high resolution of the genetic trait loci based on a dense marker map. We have shown that models of high resolution LD mappings using two flanking markers have higher power than that of models of using only one marker. Hence, high resolution LD mappings have the advantages to do fine gene mappings, and appropriate for the dense marker maps such as SNPs on human genome. Performing both LD mapping and linkage analysis has potential to avoid false positives due to population history or environmental effects. In the meantime, it takes the advantage of high resolution of $\mathrm{LD}$ mapping.

The power of association study depends on the existence of LD between trait locus and markers. In the absence of $\mathrm{LD}$, the power of $\mathrm{LD}$ mappings is very low. To increase the probability of detecting LD, one may need to carry out suitable design for a genetic study. ${ }^{24}$ It is well known that the level of LD is heavily affected by population stratification. On the one hand, the family based methods are less likely influenced by population stratification than those of population data based methods. On the other hand, a family based association study is less powerful than that of population based study for the same number of individuals. Combining the family and population data, one may expect more information, and take the advantage of population data and family data. More investigation is needed to explore the population stratification effect on high resolution LD mapping of QTL, and to develop robust methods to identify association between multiple markers and QTL in the presence of population stratification.

To our knowledge, there is not much research on statistical analysis about high resolution LD mapping of QTL. Using only one bi-allelic marker, the statistical analysis of LD mapping has been studied by a few colleagues. ${ }^{8-13}$ Relatively, multipoint linkage mapping has been studied more intensively. ${ }^{16,19,25}$ It is our hope that the current research may shed more light on the high resolution association study, and stimulate more interests to utilize the advantage of LD mapping in fine resolution of genetic studies. In the Section of power calculation and comparison, we mainly explore a set of scenarios of LD mapping. For several sets of parameters, we compare the power of four test statistics 
for LD mapping. Moreover, we compare the power of LD mapping of using population data and family data. We also investigate the effect of mutation age on the power. For linkage mapping, we only include one figure to make power comparison of linkage interval mapping using two markers with linkage mapping using only one marker. ${ }^{9}$ This reflects the need for more research on high resolution LD mapping of QTL, since the research on linkage interval/multipoint mapping is more mature.

In this paper, we treat $\mathrm{LD}$ as a fixed effect since only two markers are considered. In general, inference about the LD structure in the population are desirable, and LD should be modeled as a random effect when multiple markers/haplotypes are used in analysis, which would need more investigation. We assume that the data of all family members are available. For some late-onset diseases, the data for the parents or former family members may no longer be available. In principle, one can use similar methods as the ones proposed in this paper to perform high resolution LD mapping for sib-pair data of late-onset diseases. This is an area which is of importance and needs more research. Due to the length of this paper, we do not pursue these issues in depth, and they will be explored in other projects.

\section{Acknowledgements}

We thank two reviewers, and Dr Gert-Jan B van Ommen for their helpful comments to improve the paper. $R$ Fan was supported partially by a research fellowship from the Alexander von Humboldt Foundation, Germany, and an International Research Travel Assistance Grant of the Texas A\&M University. M Xiong was supported by NIH grant R01-GM56515, and MH59518. Ms JS Jung kindly writes the SAS micro, which is available from $R$ Fan upon request.

\section{References}

1 Botstein D, White RL, Skolnick MH, Davis RW: Construction of a genetic linkage map in man using restriction fragment length polymorphisms. Am J Hum Genet 1980; 32: 314-331.

2 Morton NE: Sequential tests for the detection of linkage. Am J Hum Genet 1955; 7: 277 -318.

3 Morton NE: Significance levels in complex inheritance. Am J Hum Genet 1998; 62: 690-697.
4 The International SNP Map Working Group: A map of human genome sequence variation containing 1.42 million single nucleotide polymorphisms. Nature 2001; 409: 928-933.

5 Risch N, Merikangas K: The future of genetic studies of complex human diseases. Science 1996; 273: 1516-1517.

6 Abecasis GR, Cherny SS, Cookson WOC, Cardon LR: Merlin rapid analysis of dense genetic maps using sparse gene flow tress. Nature Genetics 2002; 30: 97-101.

7 Almasy L, Williams JT, Dyer TD, Blangero J: Quantitative trait locus detection using combined linkage/disequilibrium analysis. Genetic Epidemiology 1999; 17 (Suppl 1): S31-S36.

8 Fulker DW, Cherny SS, Sham PC, Hewitt JK: Combined linkage and association sib-pair analysis for quantitative traits. Am J Hum Genet 1999; 64: 259-267.

9 Sham PC, Cherny SS, Purcell S, Hewitt JK: Power of linkage versus association analysis of quantitative traits, by use of variancecomponents models, for sibship data. Am J Hum Genet 2000; 66 : $1616-1630$

10 Abecasis GR, Cardon LR, Cookson WOC: A general test of association for quantitative traits in nuclear families. Am J Hum Genet 2000; 66: 279-292.

11 Abecasis GR, Cookson WOC, Cardon LR: Pedigree tests of linkage disequilibrium. Eur J Hum Genet 2000; 8: 545-551.

12 Abecasis GR, Cookson WOC, Cardon LR: The power to detect linkage disequilibrium with quantitative traits in selected samples. Am J Hum Genet 2001; 68: 1463-1474.

13 Cardon LR: A sib-pair regression model of linkage disequilibrium for quantitative traits. Hum Hered 2000; 50: 350-358.

14 Fan R, Xiong M: High resolution mapping of quantitative trait loci by linkage disequilibrium analysis. Eur J Hum Gen 2002; 10 : $607-615$.

15 Falconer DS, Mackay TFC: Introduction to Quantitative Genetics. London: Longman, 1996, 4th edn.

16 Fulker DW, Cardon LR: A sib-pair approach to interval mapping of quantitative trait loci. Am J Hum Genet 1994; 54: 1092-1103.

17 Fan R: Interval mapping of quantitative trait loci. $2002 \mathrm{http} / / /$ stat.tamu.edu/ rfan/paper.html/interval_mapping.pdf

18 Lange K: Mathematical and Statistical Methods for Genetic Analysis. New York: Springer-Verlag, 1997.

19 Almasy L, Blangero J: Multipoint quantitative trait linkage analysis in general pedigrees. Am J Hum Genet 1998; 62: 1198-1211.

20 Stuart A, Ord JK: Kendall's. Advanced Theory of Statistics, Vol. 2 Classical Inference and Relationships. Oxford, 1991, 5th edn.

21 Graybill FA: Theory and Application of the Linear Model. California: Pacific Grove, 1976.

22 Haseman JK, Elston RC: The investigation of linkage between a quantitative trait and a marker locus. Behavior Genetics 1972; 2 3-19.

23 Hartl DL, Clark AG: Principles of Population Genetics. 2nd edn. Sinauer, 1989.

24 Boehnke M, Langefeld CD: Genetic association mapping based on discordant sib pairs: the discordant-alleles test. Am J Hum Genet 1998; 62: 950-961.

25 Pratt SC, Daly M, Kruglyak: Exact multipoint quantitative-trait linkage analysis in pedigrees by variance components. $\mathrm{Am} J$ Hum Genet 2000; 66: 1153-1157. 


\section{Appendix A}

In this Appendix, we show equation (6). Actually, we have

$$
\begin{aligned}
& \mathrm{E}\left[x_{A f} x_{A 1}\right]=2 P_{a} \mathrm{E}\left[x_{A 1}, A_{f}=A A\right]+\left(\begin{array}{ll}
P_{a} & P_{A}
\end{array}\right) \mathrm{E}\left[x_{A 1}, A_{f}=A a\right] \\
& 2 P_{A} \mathrm{E}\left[x_{A 1}, A_{f}=a a\right] \\
& =2 P_{a}\left[2 P_{a} P_{A}+\left(\begin{array}{lll}
P_{a} & P_{A}
\end{array}\right) P_{a} \quad 2 P_{A} \cdot 0\right] P_{A}^{2} \\
& \left.2 P_{A}\left[\begin{array}{lll}
2 P_{a} \cdot 0+\left(P_{a}\right. & P_{A}
\end{array}\right) P_{A} \quad 2 P_{A} P_{a}\right] P_{a}^{2} \\
& +\left(\begin{array}{ll}
P_{a} & P_{A}
\end{array}\right)\left[2 P_{a} P_{A}+\left(\begin{array}{ll}
P_{a} & P_{A}
\end{array}\right)\left(P_{a}+P_{A}\right) \quad 2 P_{A} P_{a}\right] \\
& \left(2 P_{A} P_{a} / 2\right) \\
& =2 P_{a} P_{a} P_{A}^{2}+\left(\begin{array}{ll}
P_{a} & P_{A}
\end{array}\right)\left(\begin{array}{ll}
P_{a} & P_{A}
\end{array}\right) P_{a} P_{A} \\
& 2 P_{A}\left(P_{A} P_{a}^{2}\right)=P_{a} P_{A}
\end{aligned}
$$

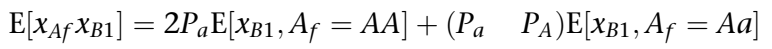

$$
\begin{aligned}
& 2 P_{A} \mathrm{E}\left[x_{B 1}, A_{f}=a a\right] \\
& =2 P_{a}\left[2 P_{b} \cdot P(A B) P_{A} \cdot P_{B}+\left(\begin{array}{ll}
P_{b} & P_{B}
\end{array}\right) .\right. \\
& \left.\left(P(A B) P_{A} \cdot P_{b}+P(A b) P_{A} \cdot P_{B}\right) 2 P_{B} \cdot P(A b) P_{A} \cdot P_{b}\right] \\
& +\left(\begin{array}{ll}
P_{a} & P_{A}
\end{array}\right)\left[2 P_{b} \cdot\left(P(A B) P_{a} \cdot P_{B}+P(a B) P_{A} \cdot P_{B}\right)\right. \\
& +\left(\begin{array}{ll}
P_{b} & P_{B}
\end{array}\right) \cdot\left(P(A B) P_{a} \cdot P_{b}+P(A b) P_{a} \cdot P_{B}\right. \\
& \left.+P(a B) P_{A} \cdot P_{b}+P(a b) P_{A} \cdot P_{B}\right) \\
& \left.2 P_{B} \cdot\left(P(A b) P_{a} \cdot P_{b}+P(a b) P_{A} \cdot P_{b}\right)\right] \\
& 2 P_{A}\left[2 P_{b} \cdot P(a B) P_{a} \cdot P_{B}\right. \\
& +\left(\begin{array}{ll}
P_{b} & P_{B}
\end{array}\right) \cdot\left(P(a B) P_{a} \cdot P_{b}+P(a b) P_{a} \cdot P_{B}\right) \\
& \left.2 P_{B} \cdot P(a b) P_{a} \cdot P_{b}\right] \\
& =2 P_{a}\left[P(A B) P_{A} P_{b} \quad P(A b) P_{A} P_{B}\right] \\
& +\left(\begin{array}{ll}
P_{a} & P_{A}
\end{array}\right)\left[P(A B) P_{a} P_{b}+P(a B) P_{A} P_{b}\right. \\
& \left.P(A b) P_{a} P_{B} \quad P(a b) P_{A} P_{B}\right] \quad 2 P_{A}\left[P(a B) P_{a} P_{b}\right. \\
& \left.P(a b) P_{a} P_{B}\right] \\
& =P(A B) P_{a} P_{b} \quad P(a B) P_{A} P_{b} \quad P(A b) P_{a} P_{B} \\
& +P(a b) P_{A} P_{B}=D_{A B} \\
& \mathrm{E}\left[\chi_{A f} Z_{A 1}\right]=2 P_{a} \mathrm{E}\left[z_{A 1}, A_{f}=A A\right]+\left(\begin{array}{ll}
P_{a} & P_{A}
\end{array}\right) \mathrm{E}\left[z_{A 1}, A_{f}=A a\right] \\
& 2 P_{A} \mathrm{E}\left[z_{A 1}, A_{f}=a a\right] \\
& =2 P_{a}\left[\begin{array}{ll}
P_{a}^{2} P_{A}+\left(P_{a} P_{A}\right) P_{a} & P_{A}^{2} \cdot 0
\end{array}\right] P_{A}^{2} \\
& 2 P_{A}\left[\begin{array}{ll}
P_{a}^{2} \cdot 0+P_{a} P_{A} P_{A} & P_{A}^{2} P_{a}
\end{array}\right] P_{a}^{2} \\
& +\left(\begin{array}{ll}
P_{a} & P_{A}
\end{array}\right)\left[P_{a}^{2} P_{A}+P_{a} P_{A}\left(P_{a}+P_{A}\right)\right. \\
& \left.P_{A}^{2} P_{a}\right] 2 P_{A} P_{a} / 2=0
\end{aligned}
$$

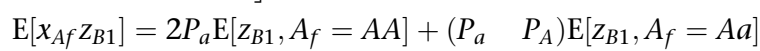

$$
\begin{aligned}
& 2 P_{A} \mathrm{E}\left[z_{B 1}, A_{f}=a a\right] \\
& =2 P_{a}\left[P_{b}^{2} \cdot P(A B) P_{A} \cdot P_{B}+P_{b} P_{B}\right. \text {. }
\end{aligned}
$$

$$
\begin{aligned}
& \left.\left(P(A B) P_{A} \cdot P_{b}+P(A b) P_{A} \cdot P_{B}\right) \quad P_{B}^{2} \cdot P(A b) P_{A} \cdot P_{b}\right] \\
& +\left(\begin{array}{ll}
P_{a} & P_{A}
\end{array}\right)\left[P_{b}^{2} \cdot\left(P(A B) P_{a} \cdot P_{B}+P(a B) P_{A} \cdot P_{B}\right)\right. \\
& +P_{b} P_{B} \cdot\left(P(A B) P_{a} \cdot P_{b}+P(A b) P_{a} \cdot P_{B}+P(a B) P_{A} \cdot P_{b}\right. \\
& \left.\left.+P(a b) P_{A} \cdot P_{B}\right) \quad P_{B}^{2} \cdot\left(P(A b) P_{a} \cdot P_{b}+P(a b) P_{A} \cdot P_{b}\right)\right] \\
& 2 P_{A}\left[P_{b}^{2} \cdot P(a B) P_{a} \cdot P_{B}\right. \\
& +P_{b} P_{B} \cdot\left(P(a B) P_{a} \cdot P_{b}+P(a b) P_{a} \cdot P_{B}\right) \\
& \left.P_{B}^{2} \cdot P(a b) P_{a} \cdot P_{b}\right]=0 .
\end{aligned}
$$

Similarly, we may show the other terms in equation (6).

\section{Appendix B}

By equations (6), (7), and large number theory, we can show the approximation (8). For instance, the approximation for element on the second row and the second column is

$$
\begin{aligned}
& \frac{1}{m} \sum_{i=m+1}^{n+m}\left(\begin{array}{lll}
x_{A f i} & x_{A m i} & x_{A 1 i}
\end{array}\right) \Sigma_{i}{ }^{1}\left(\begin{array}{lll}
x_{A f i} & x_{A m i} & \left.x_{A 1 i}\right)^{\tau}
\end{array}\right.
\end{aligned}
$$

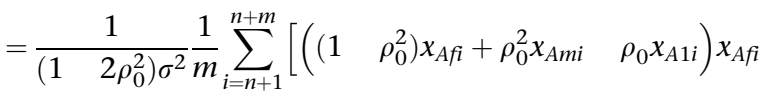

$$
\begin{aligned}
& +\left(\begin{array}{ll}
\rho_{0}^{2} x_{A f i}+\left(\begin{array}{ll}
1 & \rho_{0}^{2}
\end{array}\right) x_{A m i} & \rho_{0} x_{A 1 i}
\end{array}\right) x_{A m i} \\
& +\left(\begin{array}{cc}
\rho_{0} x_{A f i} & \left.\rho_{0} x_{A m i}+x_{A 1 i}\right) x_{A 1 i}
\end{array}\right] \\
& \left.\approx \frac{1}{\left(1 \quad 2 \rho_{0}^{2}\right) \sigma^{2}}\left[\begin{array}{ll}
2(1 & \rho_{0}^{2}
\end{array}\right) 2 P_{a} P_{A} \quad 4 \rho_{0} P_{a} P_{A}+2 P_{a} P_{A}\right] \\
& =\frac{2 P_{a} P_{A}\left(3 \quad 2 \rho_{0} \quad 2 \rho_{0}^{2}\right.}{)} \text {. }
\end{aligned}
$$

For the element on the forth row and the forth column, we have

$$
\begin{aligned}
& \left.\frac{1}{m} \sum_{i=m+1}^{n+m}\left(\begin{array}{llllll}
z_{A f i} & Z_{A m i} & Z_{A 1 i}
\end{array}\right) \Sigma_{i}{ }^{1} \quad Z_{A f i} \quad Z_{A m i} \quad Z_{A 1 i}\right)^{\tau}
\end{aligned}
$$

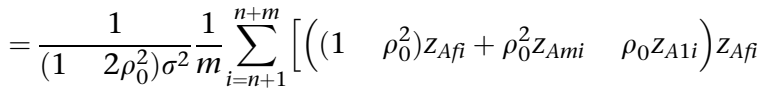

$$
\begin{aligned}
& +\left(\begin{array}{ll}
\rho_{0}^{2} z_{A f i}+\left(\begin{array}{ll}
1 & \rho_{0}^{2}
\end{array}\right) z_{A m i} & \rho_{0} z_{A 1 i}
\end{array}\right) z_{A m i}
\end{aligned}
$$

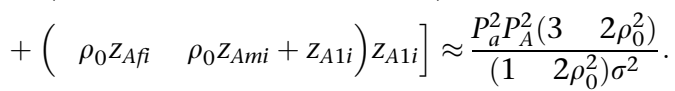




\section{Appendix C}

To prove equation (10), we first have

$$
\begin{aligned}
\mathrm{E}\left(x_{A 1} x_{A 2}\right)= & P_{A}^{4} \mathrm{E}\left[x_{A 1} x_{A 2} \mid A_{f}=A A, A_{m}=A A\right] \\
& +2 P_{A}^{2}\left(2 P_{A} P_{a}\right) \mathrm{E}\left[x_{A 1} x_{A 2} \mid A_{f}=A A, A_{m}=A a\right] \\
& +2 P_{A}^{2} P_{a}^{2} \mathrm{E}\left[x_{A 1} x_{A 2} \mid A_{f}=A A, A_{m}=a a\right] \\
& +\left(2 P_{A} P_{a}\right)^{2} \mathrm{E}\left[x_{A 1} x_{A 2} \mid A_{f}=A a, A_{m}=A a\right] \\
& +2 P_{a}^{2}\left(2 P_{A} P_{a}\right) \mathrm{E}\left[x_{A 1} x_{A 2} \mid A_{f}=A a, A_{m}=a a\right] \\
& +P_{a}^{4} \mathrm{E}\left[x_{A 1} x_{A 2} \mid A_{f}=a a, A_{m}=a a\right] \\
= & P_{A}^{4}\left(4 P_{a}^{2}\right)+4 P_{A}^{3} P_{a}\left[2 P_{a} / 2+\left(P_{a} \quad P_{A}\right) / 2\right]^{2} \\
& +2 P_{A}^{2} P_{a}^{2}\left[P_{a} \quad P_{A}\right]^{2}+\left(2 P_{A} P_{a}\right)^{2} . \\
& {\left[P_{a} / 4+\left(P_{a} \quad P_{A}\right) / 2 \quad 2 P_{A} / 4\right]^{2} } \\
& +4 P_{a}^{3} P_{A}\left[\left(P_{a} \quad P_{A}\right) / 2 \quad 2 P_{A} / 2\right]^{2}+P_{a}^{4}\left(4 P_{A}^{2}\right) \\
= & P_{A} P_{a} \\
\mathrm{E}\left(x_{A 1} Z_{A 2}\right)= & P_{A}^{4} \mathrm{E}\left[x_{A 1} Z_{A 2} \mid A_{f}=A A, A_{m}=A A\right] \\
& +2 P_{A}^{2}\left(2 P_{A} P_{a}\right) \mathrm{E}\left[x_{A 1} Z_{A 2} \mid A_{f}=A A, A_{m}=A a\right] \\
& +2 P_{A}^{2} P_{a}^{2} \mathrm{E}\left[x_{A 1} Z_{A 2} \mid A_{f}=A A, A_{m}=a a\right] \\
& +\left(2 P_{A} P_{a}\right)^{2} \mathrm{E}\left[x_{A 1} Z_{A 2} \mid A_{f}=A a, A_{m}=A a\right] \\
& +2 P_{a}^{2}\left(2 P_{A} P_{a}\right) \mathrm{E}\left[x_{A 1} Z_{A 2} \mid A_{f}=A a, A_{m}=a a\right] \\
& +P_{a}^{4} \mathrm{E}\left[x_{A 1} Z_{A 2} \mid A_{f}=a a, A_{m}=a a\right] \\
= & P_{A}^{4}\left(2 P_{a}^{3}\right)+4 P_{A}^{3} P_{a}\left[2 P_{a} / 2+\left(P_{a} \quad P_{A}\right) / 2\right] \\
& {\left[P_{a}^{2} / 2+P_{a} P_{A} / 2\right]+2 P_{A}^{2} P_{a}^{2}\left[P_{a} \quad P_{A}\right] P_{a} P_{A} } \\
& +\left(2 P_{A} P_{a}\right)^{2}\left[2 P_{a} / 4+\left(P_{a} \quad P_{A}\right) / 2 \quad 2 P_{A} / 4\right] \\
& {\left[P_{a}^{2} / 4+P_{a} P_{A} / 2 \quad P_{A}^{2} / 4\right] } \\
& +4 P_{a}^{3} P_{A}\left[\left(P_{a} \quad P_{A}\right) / 2 \quad 2 P_{A} / 2\right]\left[P_{a} P_{A} / 2 \quad P_{A}^{2} / 2\right] \\
& +P_{a}^{4}\left(2 P_{A}^{3}\right)=0 \\
& 0 \\
&
\end{aligned}
$$

$$
\begin{aligned}
\mathrm{E}\left(z_{A 1} z_{A 2}\right)= & P_{A}^{4} \mathrm{E}\left[z_{A 1} z_{A 2} \mid A_{f}=A A, A_{m}=A A\right] \\
& +2 P_{A}^{2}\left(2 P_{A} P_{a}\right) \mathrm{E}\left[z_{A 1} z_{A 2} \mid A_{f}=A A, A_{m}=A a\right] \\
& +2 P_{A}^{2} P_{a}^{2} \mathrm{E}\left[z_{A 1} z_{A 2} \mid A_{f}=A A, A_{m}=a a\right] \\
& +\left(2 P_{A} P_{a}\right)^{2} \mathrm{E}\left[z_{A 1} z_{A 2} \mid A_{f}=A a, A_{m}=A a\right] \\
& +2 P_{a}^{2}\left(2 P_{A} P_{a}\right) \mathrm{E}\left[z_{A 1} z_{A 2} \mid A_{f}=A a, A_{m}=a a\right] \\
& +P_{a}^{4} \mathrm{E}\left[z_{A 1} z_{A 2} \mid A_{f}=a a, A_{m}=a a\right] \\
= & P_{A}^{4}\left(P_{a}^{2}\right)^{2}+4 P_{A}^{3} P_{a}\left[P_{a}^{2} / 2+P_{a} P_{A} / 2\right]^{2} \\
& +2 P_{A}^{2} P_{a}^{2}\left[P_{a} P_{A}\right]^{2} \\
& +\left(2 P_{A} P_{a}\right)^{2}\left[P_{a}^{2} / 4+P_{a} P_{A} / 2 \quad P_{A}^{2} / 4\right]^{2} \\
& +4 P_{a}^{3} P_{A}\left[P_{a} P_{A} / 2 \quad P_{A}^{2} / 2\right]^{2}+P_{a}^{4}\left(P_{A}^{2}\right)^{2} \\
= & P_{a}^{2} P_{A}^{2} / 4 .
\end{aligned}
$$

Similarly, we may show that $\mathrm{E}\left(x_{B 1} x_{B 2}\right)=P_{b} P_{B}, \mathrm{E}\left(x_{B 1} Z_{B 2}\right)=$ $0, \mathrm{E}\left(z_{B 1} Z_{B 2}\right)=P_{b}^{2} P_{B}^{2} / 4$. To show the other terms of (10), we first calculate the joint probabilities $P\left(A_{1}, B_{2}\right)$, in which the first offspring's genotype is $A_{1}$ at marker $A$ and the second offspring's genotype is $B_{2}$ at marker $B, A_{1} \in\{A A, A a, a a\}$, $B_{2} \in\{B B, B b, b b\}$.We need to consider nine possible phases $\{A A, A a, a a\} \times\{B B, B b, b b\}$ for each parent. At the first glance, one needs to consider $9 \times 9$ possible matings to calculate $P\left(A_{1}, B_{2}\right)$. However, many matings do not lead to specific genotypes $\left(A_{1}, B_{2}\right)$ of a sib pair. This eliminates many terms and reduces the amount of calculations. For instance, a mating of $\left(A_{f}=A A, B_{f}=B B\right) \times\left(A_{m}=A A, B_{m}=B B\right)$ only results offspring with genotype $(A A, B B)$. Then, we have

$$
\begin{aligned}
& P\left(A_{1}=A A, B_{2}=B B\right) \\
= & \sum_{A_{f}, B_{f}} P\left(A_{f}, B_{f}\right) \sum_{A_{m}, B_{m}} P\left(A_{m}, B_{m}\right) P\left[A_{1}=A A, B_{2}\right. \\
= & \left.B B \mid\left(A_{f}, B_{f}\right),\left(A_{m}, B_{m}\right)\right] \\
= & P(A B)^{2}\left[P(A B)^{2}+2 \cdot 2 P(A B) P(A b) / 2+2 \cdot 2 P(A B) P(a B) / 2\right. \\
& +2 \cdot[2 P(A B) P(a b)+2 P(A b) P(a B)] / 4] \\
& +2 P(A B) P(A b)[2 P(A B) P(A b) / 4 \\
& +2 \cdot 2 P(A B) P(a B) / 4+2 \cdot[2 P(A B) P(a b) \\
& +2 P(A b) P(a B)] \cdot 1 / 2 \cdot 1 / 4] \\
& +2 P(A B) P(a B)[2 P(A B) P(a B) / 4+2 \cdot[2 P(A B) P(a b) \\
& +2 P(A b) P(a B)] \cdot 1 / 2 \cdot 1 / 4] \\
& +[2 P(A B) P(a b)+2 P(A b) P(a B)]^{2} \cdot 1 / 4 \cdot 1 / 4 \\
= & \left(P(A B) \quad P_{A} P_{B}\right)^{2} / 4+P_{A} P_{B} P(A B)=\Delta_{A B}^{2} / 4+P_{A} P_{B} P(A B) .
\end{aligned}
$$

Symmetrically, we may get the following three terms

$$
\begin{gathered}
P\left(A_{1}=A A, B_{2}=b b\right)=\Delta_{A B}^{2} / 4+P_{A} P_{b} P(A b), \\
P\left(A_{1}=a a, B_{2}=B B\right)=\Delta_{A B}^{2} / 4+P_{a} P_{B} P(a B), \\
P\left(A_{1}=a a, B_{2}=b b\right)=\Delta_{A B}^{2} / 4+P_{a} P_{b} P(a b) .
\end{gathered}
$$

Note that $P\left(A_{1}=A A, B_{2}=B b\right)=P\left(A_{1}=A A\right)-P\left(A_{1}=A A, B_{2}=B B\right.$ or $b b)$. Hence,

$$
P\left(A_{1}=A A, B_{2}=B b\right)=\Delta_{A B}^{2} / 2+P(A B) P_{A} P_{b}+P(A b) P_{A} P_{B} .
$$

Similarly, we may calculate the following three terms

$$
\begin{array}{cl}
P\left(A_{1}=a a, B_{2}=B b\right)= & \Delta_{A B}^{2} / 2+P(a B) P_{a} P_{b}+P(a b) P_{a} P_{B} \\
P\left(A_{1}=A a, B_{2}=B B\right)= & \Delta_{A B}^{2} / 2+P(A B) P_{a} P_{B}+P(a B) P_{A} P_{B} \\
P\left(A_{1}=A a, B_{2}=b b\right)= & \Delta_{A B}^{2} / 2+P(A b) P_{a} P_{b}+P(a b) P_{A} P_{b} .
\end{array}
$$

Finally, we can calculate the following term using equation $\Sigma_{\mathrm{A} 1} \Sigma_{B 2} P\left(A_{1}, B_{2}\right)=1$

$$
\begin{aligned}
& P\left(A_{1}=A a, B_{2}=B b\right) \\
& =\Delta_{A B}^{2}+P(A B) P_{a} P_{b}+P(A b) P_{a} P_{B}+P(a B) P_{A} P_{b}+P(a b) P_{A} P_{B} .
\end{aligned}
$$


Using equations (16), (17), (18), (19) and (20), we may calculate

$$
\begin{aligned}
\mathrm{E}\left[\chi_{A 1} \chi_{B 2}\right]=2 & P_{a}\left[2 P_{b} P\left[A_{1}=A A, B_{2}=B B\right]\right. \\
+ & \left(P_{b} \quad P_{B}\right) P\left[A_{1}=A A, B_{2}=B b\right] \\
& \left.2 P_{B} P\left[A_{1}=A A, B_{2}=b b\right]\right] \\
+ & \left(P_{a} \quad P_{A}\right)\left[2 P_{b} P\left[A_{1}=A a, B_{2}=B B\right]\right. \\
+ & \left(P_{b} \quad P_{B}\right) P\left[A_{1}=A a, B_{2}=B b\right] \\
& \left.2 P_{B} P\left[A_{1}=A a, B_{2}=b b\right]\right] \\
& 2 P_{A}\left[2 P_{b} P\left[A_{1}=a a, B_{2}=B B\right]\right. \\
+ & \left(P_{b} \quad P_{B}\right) P\left[A_{1}=a a, B_{2}=B b\right] \\
& \left.2 P_{B} P\left[A_{1}=a a, B_{2}=b b\right]\right]=D_{A B} .
\end{aligned}
$$

Similarly, we may get $\mathrm{E}\left[x_{A 1} z_{B 2}\right]=0, \mathrm{E}\left[z_{A 1} z_{B 2}\right]=D_{A B}^{2} / 4$. By symmetric property, we may calculate the remaining terms in (10).

\section{Appendix D}

Let $\sum_{i}{ }^{1}$ be the matrix given by (9). To show the approximation of (11), we notice that $d_{11}$ can be calculated by $d_{11}=\sigma^{2}\left(\begin{array}{llll}1 & 1 & 1 & 1\end{array}\right) \sum_{i}^{1}\left(\begin{array}{llll}1 & 1 & 1 & 1\end{array}\right)^{\tau}$. The element on the second row and the second column of approximation (11) can be calculated by

$$
\begin{aligned}
& \frac{1}{k} \sum_{i=n+m+1}^{n+m+k}\left(x_{A f i} \quad x_{A m i} \quad x_{A 1 i} \quad x_{A 2 i}\right) \Sigma_{i}^{1}\left(\begin{array}{llll}
x_{A f i} & x_{A m i} & x_{A 1 i} & \left.x_{A 2 i}\right)^{\tau}
\end{array}\right. \\
& =\frac{1}{\sigma^{2}} \frac{1}{k} \sum_{i=n+m+1}^{n+m+k}\left[\left(\left(1+2 C \rho_{0}\right) x_{A f i}+2 C \rho_{0} x_{A m i} \quad C x_{A 1 i} \quad C x_{A 2 i}\right) x_{A f i}\right. \\
& +\left(2 C \rho_{0} x_{A f i}+\left(1+2 C \rho_{0}\right) x_{A m i} \quad C x_{A 1 i} \quad C x_{A 2 i}\right) x_{A m i}
\end{aligned}
$$

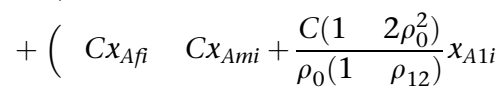

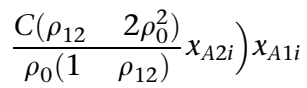

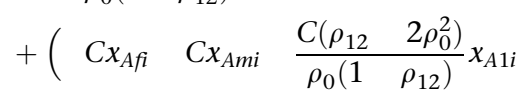

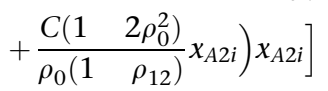

$$
\begin{aligned}
& \approx \frac{1}{\sigma^{2}}\left[\left(\left(1+2 C \rho_{0}\right) 2 P_{a} P_{A}+0 \quad C P_{a} P_{A} \quad C P_{a} P_{A}\right)\right. \\
& +\left(0+\left(1+2 C \rho_{0}\right) 2 P_{a} P_{A} \quad C P_{a} P_{A} \quad C P_{a} P_{A}\right)
\end{aligned}
$$

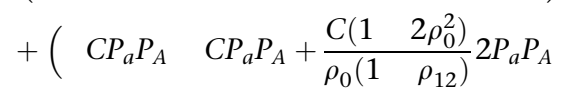

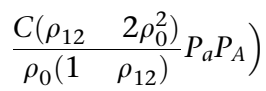

$$
\begin{aligned}
& +\left(\begin{array}{cccc}
C P_{a} P_{A} & C P_{a} P_{A} & \frac{C\left(\rho_{12}\right.}{2} 2 \rho_{0}^{2}
\end{array}\right) P_{a} P_{A}
\end{aligned}
$$

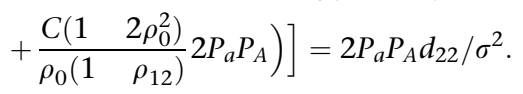

Similarly, the element on the forth row and the forth column of approximation (11) is

$$
\begin{aligned}
& \frac{1}{k} \sum_{i=n+m+1}^{n+m+k}\left(\begin{array}{lllllll}
z_{A f i} & Z_{A m i} & Z_{A 1 i} & Z_{A 2 i}
\end{array}\right) \Sigma_{i}{ }^{1}\left(\begin{array}{llll}
Z_{A f i} & Z_{A m i} & Z_{A 1 i} & \left.Z_{A 2 i}\right)^{\tau}
\end{array}\right. \\
& =\frac{1}{\sigma^{2}} \frac{1}{k} \sum_{i=n+m+1}^{n+m+k}\left[\left(\begin{array}{lll}
\left(1+2 C \rho_{0}\right) z_{A f i}+2 C \rho_{0} z_{A m i} & C z_{A 1 i} & C z_{A 2 i}
\end{array}\right) z_{A f i}\right. \\
& +\left(2 C \rho_{0} z_{A f i}+\left(1+2 C \rho_{0}\right) z_{A m i} \quad C z_{A 1 i} \quad C z_{A 2 i}\right) z_{A m i}
\end{aligned}
$$

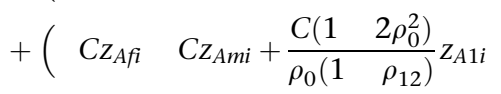

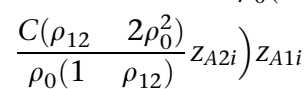

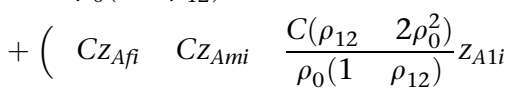

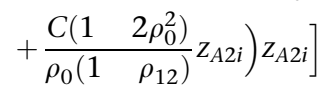

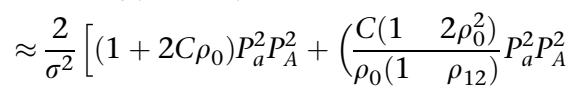

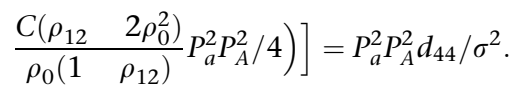

The other terms of approximation (11) can be calculated in a similar manner. 